\title{
Kruppel-like factor 4 is critical for transcriptional control of cardiac mitochondrial homeostasis
}

\author{
Xudong Liao, ${ }^{1}$ Rongli Zhang, ${ }^{1}$ Yuan Lu, ${ }^{1}$ Domenick A. Prosdocimo, ${ }^{1}$ Panjamaporn Sangwung, ${ }^{1}$ Lilei Zhang, ${ }^{1}$ Guangjin Zhou, ${ }^{1}$ \\ Puneet Anand, ${ }^{2}$ Ling Lai,, ${ }^{3}$ Teresa C. Leone, ${ }^{3}$ Hisashi Fujioka, ${ }^{4,5}$ Fang Ye, ${ }^{5}$ Mariana C. Rosca, ${ }^{6}$ Charles L. Hoppel, ${ }^{5}$ \\ P. Christian Schulze, ${ }^{7}$ E. Dale Abel, ${ }^{8}$ Jonathan S. Stamler, ${ }^{2}$ Daniel P. Kelly, ${ }^{3}$ and Mukesh K. Jain ${ }^{1}$ \\ 'Case Cardiovascular Research Institute and Institute for Transformative Molecular Medicine, Case Western Reserve University School of Medicine and University Hospitals Case Medical Center, \\ Cleveland, Ohio, USA. ${ }^{3}$ Cardiovascular Pathobiology Program, Diabetes and Obesity Research Center, Sanford-Burnham Medical Research Institute, Orlando, Florida, USA. ${ }^{4}$ Electron Microscopy Facility and \\ ${ }^{5}$ Center for Mitochondrial Diseases, Department of Pharmacology, Case Western Reserve University, Cleveland, Ohio, USA. ${ }^{6}$ Central Michigan University College of Medicine, Mount Pleasant, Michigan, USA. \\ ${ }^{7}$ Division of Cardiology, Department of Medicine, Columbia University Medical Center, New York, New York, USA. ${ }^{8}$ Fraternal Order of Eagles Diabetes Research Center and Division of Endocrinology \\ and Metabolism, University of lowa Carver College of Medicine, lowa City, lowa, USA
}

\begin{abstract}
Mitochondrial homeostasis is critical for tissue health, and mitochondrial dysfunction contributes to numerous diseases, including heart failure. Here, we have shown that the transcription factor Kruppel-like factor 4 (KLF4) governs mitochondrial biogenesis, metabolic function, dynamics, and autophagic clearance. Adult mice with cardiac-specific KIf4 deficiency developed cardiac dysfunction with aging or in response to pressure overload that was characterized by reduced myocardial ATP levels, elevated ROS, and marked alterations in mitochondrial shape, size, ultrastructure, and alignment. Evaluation of mitochondria isolated from KLF4-deficient hearts revealed a reduced respiration rate that is likely due to defects in electron transport chain complex I. Further, cardiac-specific, embryonic KIf4 deletion resulted in postnatal premature mortality, impaired mitochondrial biogenesis, and altered mitochondrial maturation. We determined that KLF4 binds to, cooperates with, and is requisite for optimal function of the estrogen-related receptor/PPAR $\gamma$ coactivator 1 (ERR/PGC-1) transcriptional regulatory module on metabolic and mitochondrial targets. Finally, we found that KLF4 regulates autophagy flux through transcriptional regulation of a broad array of autophagy genes in cardiomyocytes. Collectively, these findings identify KLF4 as a nodal transcriptional regulator of mitochondrial homeostasis.
\end{abstract}

\section{Introduction}

The heart has an unrelenting need for energy throughout life to sustain contractile function. Despite its high-energy demand, the heart contains relatively low ATP reserves, and thus, a continual supply of energy is required (1). To meet such demand, cardiomyocytes are endowed with a large mitochondrial content that accounts for almost all ( $>95 \%)$ ATP production derived from oxidative phosphorylation (OXPHOS) (2). As a robust uninterrupted ATP supply from mitochondria is critical to maintain cardiac function, it is not surprising that subtle alterations in mitochondria function can have a dramatic influence on heart function. Indeed, mitochondrial dysfunction is increasingly rec-

Note regarding evaluation of this manuscript: Manuscripts authored by scientists associated with Duke University, The University of North Carolina at Chapel Hill, Duke-NUS, and the Sanford-Burnham Medical Research Institute are handled not by members of the editorial board but rather by the science editors, who consult with selected external editors and reviewers.

Conflict of interest: Jonathan S. Stamler has ownership in Adamas Pharmaceuticals, LifeHealth LLC, and Nivalis Therapeutics; receives income from LifeHealth LLC and Novartis; and holds numerous outstanding patents and patent applications. Daniel P. Kelly is a consultant for Pfizer Inc. and receives research support from Takeda Pharmaceuticals and Acorda Therapeutics.

Submitted: November 12, 2014; Accepted: June 18, 2015.

Reference information: / Clin Invest. 2015;125(9):3461-3476. doi:10.1172/JCI79964. ognized as critical to the pathogenesis of cardiac disease (3). However, the molecular mechanisms controlling mitochondrial health remain incompletely understood.

Mitochondria are dynamic organelles, whose collective mass and morphology is regulated through mitochondrial biogenesis, dynamics (fission/fusion), and autophagic clearance (mitophagy). Developmental and physiological states associated with increased energy demand (e.g., postnatal cardiac maturation and exercise) lead to a significant increase in mitochondrial numbers through biogenesis $(4,5)$. In addition, mitochondrial dynamics help maintain mitochondrial homeostasis through repeated fusion and fission cycles in which mitochondrial contents are mixed and damaged molecules are either diluted or compensated by healthy ones as a form of complementation (6). Finally, the quality of mitochondria is maintained through mitophagy, a specific form of autophagy in which defective mitochondria are selectively isolated from the cellular network and subjected to lysosomal degradation (7). Accumulating evidence has demonstrated that perturbation of any phase of mitochondrial life cycle in the heart impairs heart function $(5,8-10)$.

Studies over the past decades support an important role for a subset of the nuclear receptor superfamily in regulation of cardiac metabolism and mitochondrial homeostasis, including the PPAR family (Ppara, Ppard, Pparg), the estrogen-related receptor (ERR) 
A

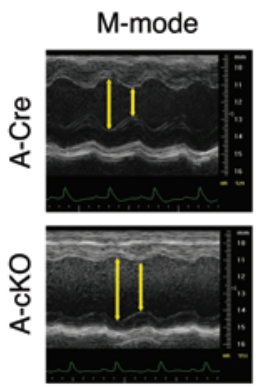

B

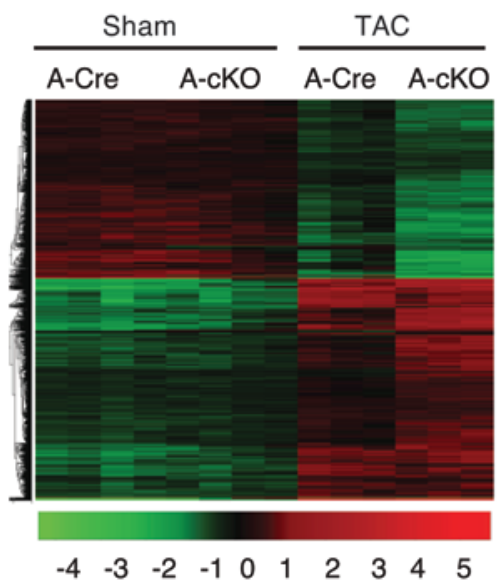

C

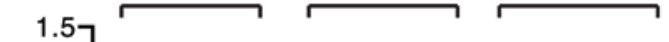
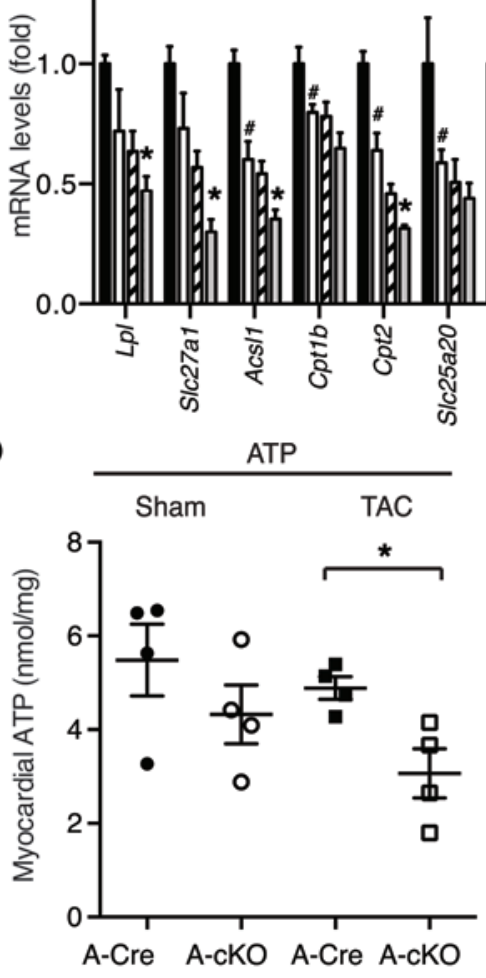

E
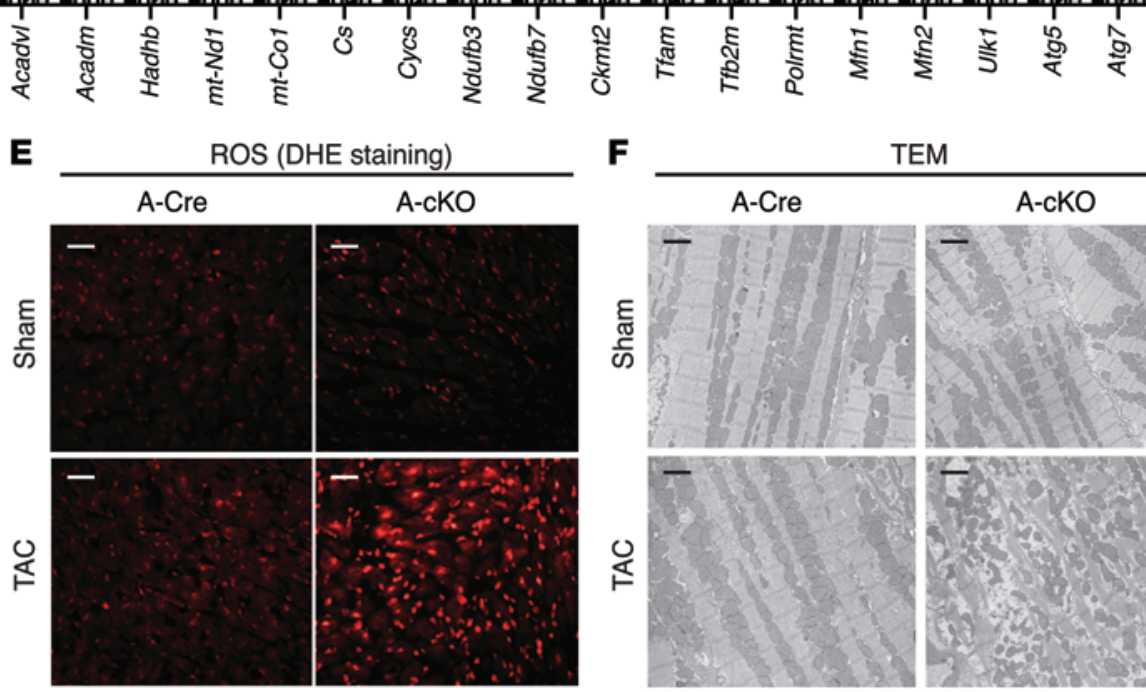

Mitochondrion (ES 5.443)

Inflammatory response (ES 2.28)

Muscle fiber (ES 3.897)

Hypertrophy (ES 2.34)

Sarcoplasmic reticulum (ES 2.74)

Heart rate (ES 1.893)

A-Cre Sham $\square$ A-cKO Sham $\square A-C r e$ TAC $\square$ A-cKO TAC OXPHOS
Biogenesis Dynamics Autophagy
$\mathbf{F}$

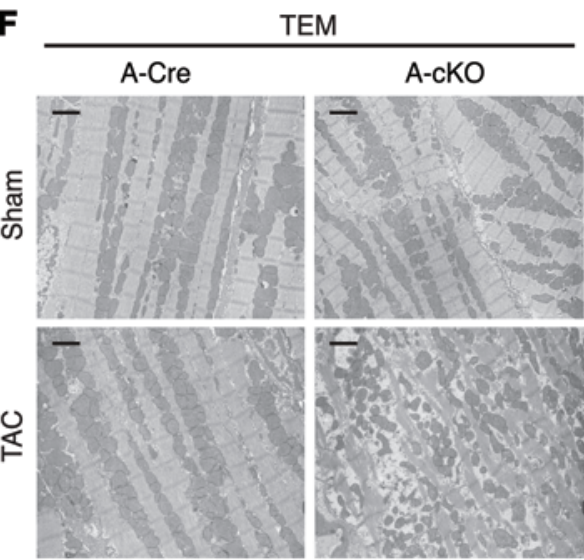


Figure 1. KLF4 is required for the bioenergetic and functional adaptation to cardiac pressure overload. (A) LV function, heart weight (HW), lung weight (LW), and expression of hypertrophic marker genes in the heart. BW, body weight. (B) Microarray analysis. Heat map showing marginal differences in sham groups but dramatic differences in TAC groups between $A-C r e$ and A-cKO mice. Genes that were differentially expressed in TAC groups (fold change over 1.5, A-cKO vs. A-Cre mice) were subjected to gene enrichment analysis. ES, enrichment score. (C) qPCR analysis of some cardiac metabolic and mitochondrial genes. $n=4$ for sham groups, $n=7$ for A-Cre TAC group, $n=5$ for A-CKO TAC group. (D) Myocardial ATP levels. (E) Myocardial ROS levels, as assessed by DHE staining. Scale bar: 20 $\mu \mathrm{m}$. (F) Myocardium ultrastructure, as assessed by transmission electron microscopy (TEM), showing mitochondrial damage after TAC. Scale bar: 2 $\mu \mathrm{m}$. Representative images $(n=3)$ are shown. Measurements were taken 3 days after sham and TAC operations. ${ }^{P} P<0.05$ between sham groups; ${ }^{*} P<0.05$ between TAC groups, Student's $t$ test with Bonferroni correction.

family (Esrra, Esrrb, Esrrg), and the PPAR $\gamma$ coactivator 1 family (e.g., Ppargc1a and Ppargc1b, also called PGC-1 $\alpha$ and PGC-1 $\beta$ ). Current paradigms suggest that the ERR/PGC-1 molecular module regulates a broad spectrum of genes that are involved in cardiac energy metabolism, mitochondrial biogenesis, dynamics, and OXPHOS (11-15). These effects are thought to occur through direct transcriptional control of key targets or indirectly through induction of effectors such as PPAR $\alpha$, a dominant regulator of cardiac fatty acid oxidation (FAO) (16). However, the role of PGC-1 signaling in autophagy remains controversial $(17,18)$. To date, studies of autophagy have largely focused on cytoplasmic events and little is known about transcriptional control of this process (19).

Kruppel-like factors (KLFs) belong to the zinc finger family of transcription factors. Studies from our group and others have identified members of this family as critical regulators of cellular metabolism and muscle function (20-22). For example, previous work has identified KLF4 as critical for the heart's adaptation to stress, but the molecular basis for this was unknown $(23,24)$. Here, we provide evidence indicating that KLF4 regulates all major aspects of mitochondrial life cycle, from metabolic function to biogenesis, dynamics, and autophagic clearance. Collectively, this study identifies KLF4 as central for transcriptional control of mitochondrial health in the heart.

\section{Results}

Cardiac Klf4 deficiency impairs myocardial function and energetics with stress. Previous work by our group showed that cardiac-specific postnatal deletion of Klf4 gene rendered mice highly susceptible to heart failure and death following pressure overload induced by transaortic constriction (TAC) (24). However, the basis for this sensitivity to stress remains unknown. To investigate this precipitous decline in cardiac function, we performed TAC in 8- to 10-week-old Myh6-Cre Klf4 ${ }^{+/+}$(designated A-Cre for adult Cre) and Myh6-Cre Klf4 $4^{f / f l}$ (designated A-cKO for adult cardiac KO) mice and assessed heart function by serial echocardiography. Consistent with the high mortality after TAC reported previously (24), A-cKO mice exhibited a marked 50\% reduction in left ventricular (LV) fractional shortening 3 days after TAC (Figure 1A). Tissue samples harvested at this time point revealed increased lung weight, suggesting pulmonary edema, and robust induction of cardiac hypertrophic markers in A-cKO mice compared with those in the A-Cre group, despite similar heart weight due to lim- ited pressure overload time (3 days) for hypertrophy (Figure 1A). Extensive biochemical and histological analysis did not reveal any alterations in myocardial structure, fibrosis, vasculature, or hypoxia in KLF4-deficient hearts. Telemetry ECG recording revealed no baseline abnormalities in A-cKO mice, but evidence for bradycardia was observed in mice with TAC-induced heart failure (Supplemental Figure 1; supplemental material available online with this article; doi:10.1172/JCI79964DS1).

In order to gain insights into the underlying molecular mechanisms, we performed unbiased microarray analysis in LV samples from A-Cre and A-cKO mice 3 days after TAC or sham operation. The gene expression profile revealed a KLF4-dependent metabolic signature, particularly in mitochondrial gene expression after TAC (Figure 1B). We confirmed this signature by quantitative RT-PCR (qPCR) analysis, and, as shown in Figure 1C, KLF4 regulated the expression of a broad array of genes involved in transcriptional regulation of FAO, OXPHOS, mitochondrial biogenesis, dynamics, and autophagy.

Consistent with the gene expression profile, we observed a significant reduction in myocardial ATP levels coupled with excessive ROS generation in A-cKO myocardium following TAC (Figure $1, \mathrm{D}$ and $\mathrm{E})$, indicating mitochondrial dysfunction. Transmission electron microscopy studies revealed striking evidence of mitochondrial damage in A-cKO myocardium following TAC, including mitochondrial disarray, degeneration, fragmentation, elongation, and increased heterogeneity (Figure $1 \mathrm{~F}$ and Supplemental Figure 2). In addition, patchy deposition of myocardial glycogen was marginally increased in the A-cKO group, which might reflect decreased mitochondrial metabolism in damaged tissue (Supplemental Figure 3).

KLF4 deficiency impairs mitochondrial function. Given these profound alterations in gene expression and mitochondrial morphology (Figure 1, C and F), we next assessed respiration in mitochondria isolated from KLF4-deficient hearts (Supplemental Table 1). These studies focused on mitochondria isolated from young control and KLF4-deficient hearts at baseline, given that pressure overload results in severe mitochondrial damage that would impede the isolation of a mitochondrial sample that is representative of the in vivo mitochondrial population. More importantly, the mitochondrial defects detectable at baseline are more likely to address a cause-effect relationship between mitochondrial dysfunction and heart failure.

Freshly isolated mitochondria from A-cKO hearts exhibited reduced respiration rates with fatty acid substrates and pyruvate (Figure 2A). As mitochondrial oxidation of fatty acids and pyruvate merges at the level of acetyl-CoA, our data suggested that KLF4 deficiency leads to a common downstream defect at either the Krebs cycle or electron transport chain (ETC) level. Indeed, direct ETC respiration studies revealed a $25 \%$ reduction in complex I function in the A-cKO group (Figure 2B), comparable to the reduction in pyruvate oxidation rate. Thus, defective complex I function likely accounts for the reduced mitochondrial OXPHOS function at baseline. In concert with the reduction in mitochondrial function, we observed an increased level of AMPK $\alpha$ phosphorylation, an indicator of AMP-high/ATP-low stage, in KLF4-deficient myocardium, indicating cellular energetic insufficiency (Figure 2C). Similar reduction in mitochondrial respiration was observed in neonatal rat 
A Palmitoyl-CoA

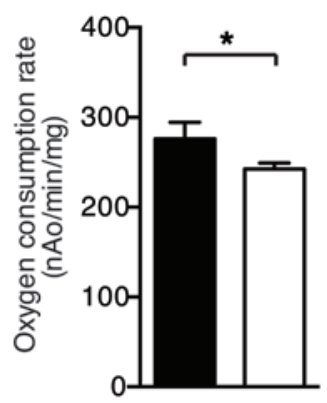

B



Palmitoyl-carnitine

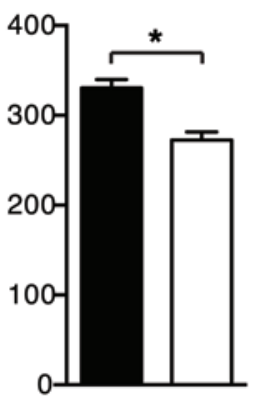

Octanoyl-carnitine

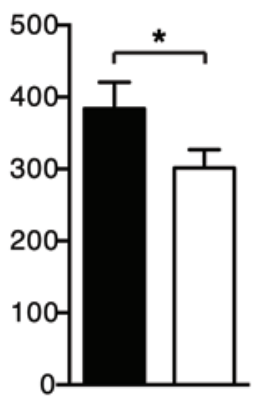

Acetyl-carnitine

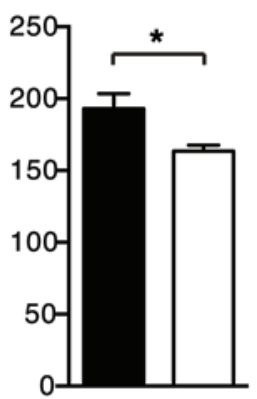

Pyruvate



III

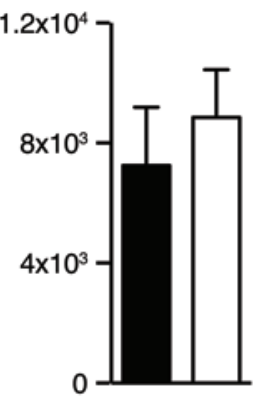

0
IV

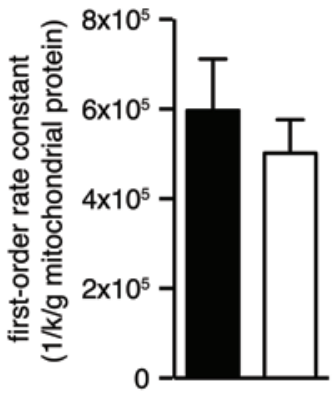

A-Cre

A-cKO

C A-Cre

A-cKO

p-AMPKa

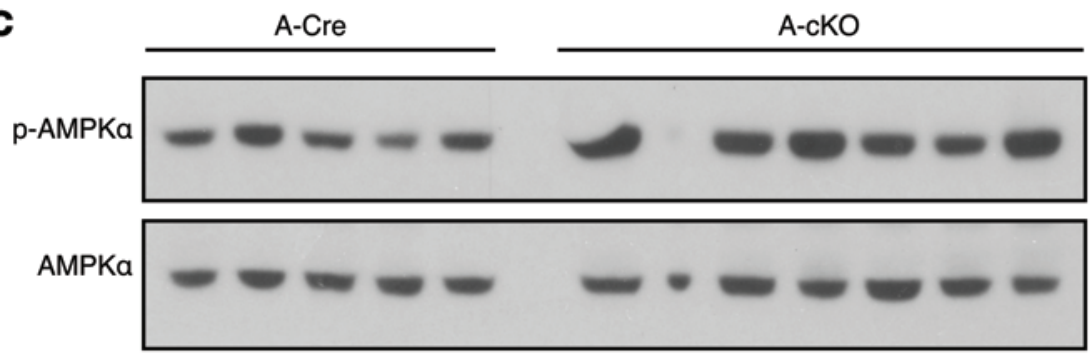

AMPKa

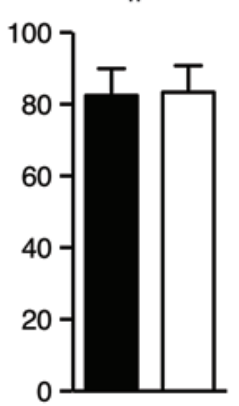

a-Tubulin
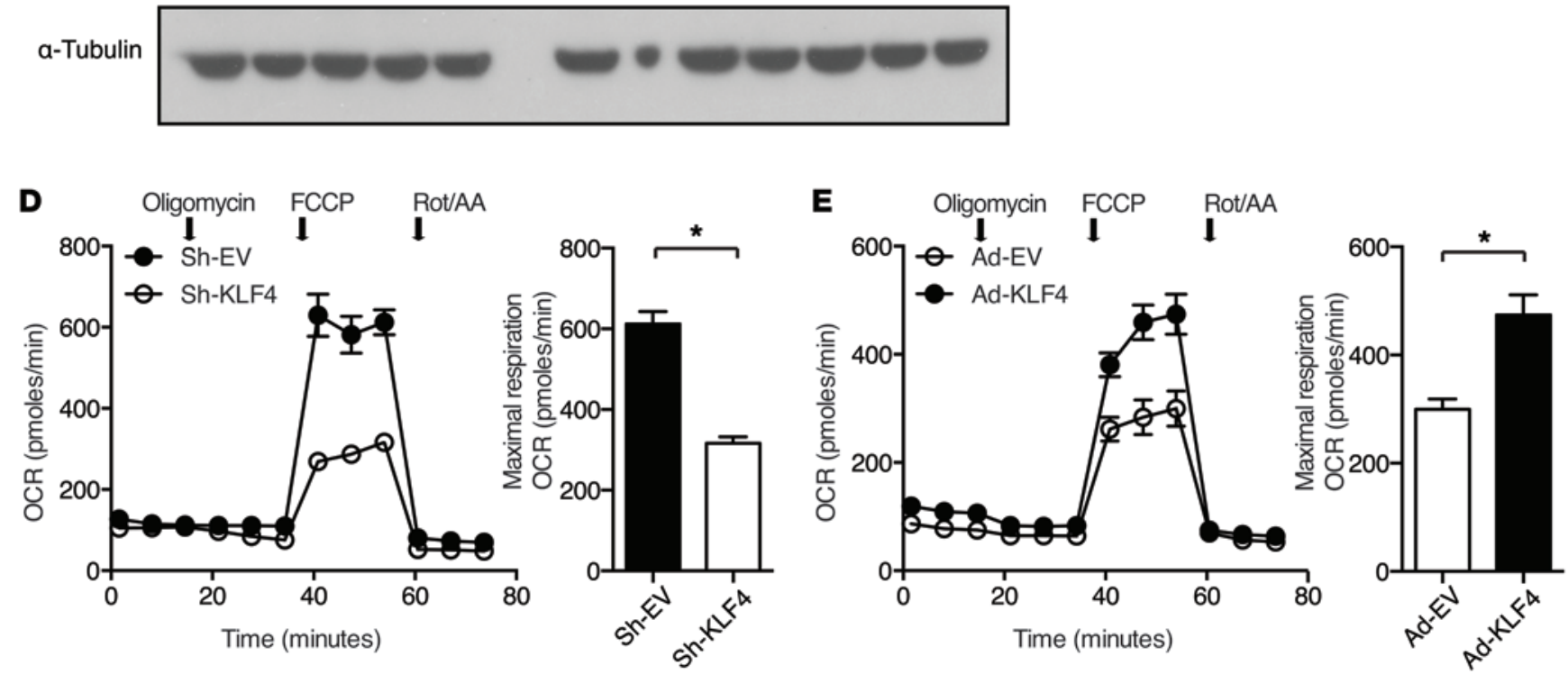

Figure 2. KLF4 deficiency impairs mitochondrial function and myocardial bioenergetics. (A) Mitochondrial respiration assays with fatty acids and pyruvate substrates. Malate was used as NADH donor. (B) Assays for ETC complex activity. Data presented are from 5 independent experiments. (C) Western blotting analysis for AMPK $\alpha$ phosphorylation. p-AMPK $\alpha, p-T h r 172$. (D and E) Cellular respiration assays with NRVMs following (D) acute adenoviral knockdown or (E) overexpression of KLF4. Glucose $(25 \mathrm{mmol} / \mathrm{I})$ and pyruvate $(1 \mathrm{mmol} / \mathrm{l})$ were supplied as substrates. OCR, oxygen consumption rate; Sh-EV, shRNA targeting empty vector; Sh-KLF4, shRNA targeting KIf4; FCCP, p-trifluoromethoxyphenylhydrazone; Rot, rotenone; $A A$, antimycin A; Ad-EV, adenoviral empty vector; Ad-KLF4, adenoviral KLF4. Maximal respiration rate equals the FCCP-induced oxygen consumption rate. $n=3 .{ }^{*} P<0.05$, Student's $t$ test. 


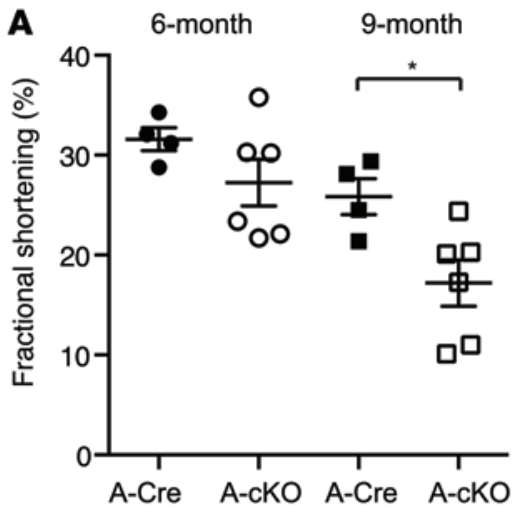

B
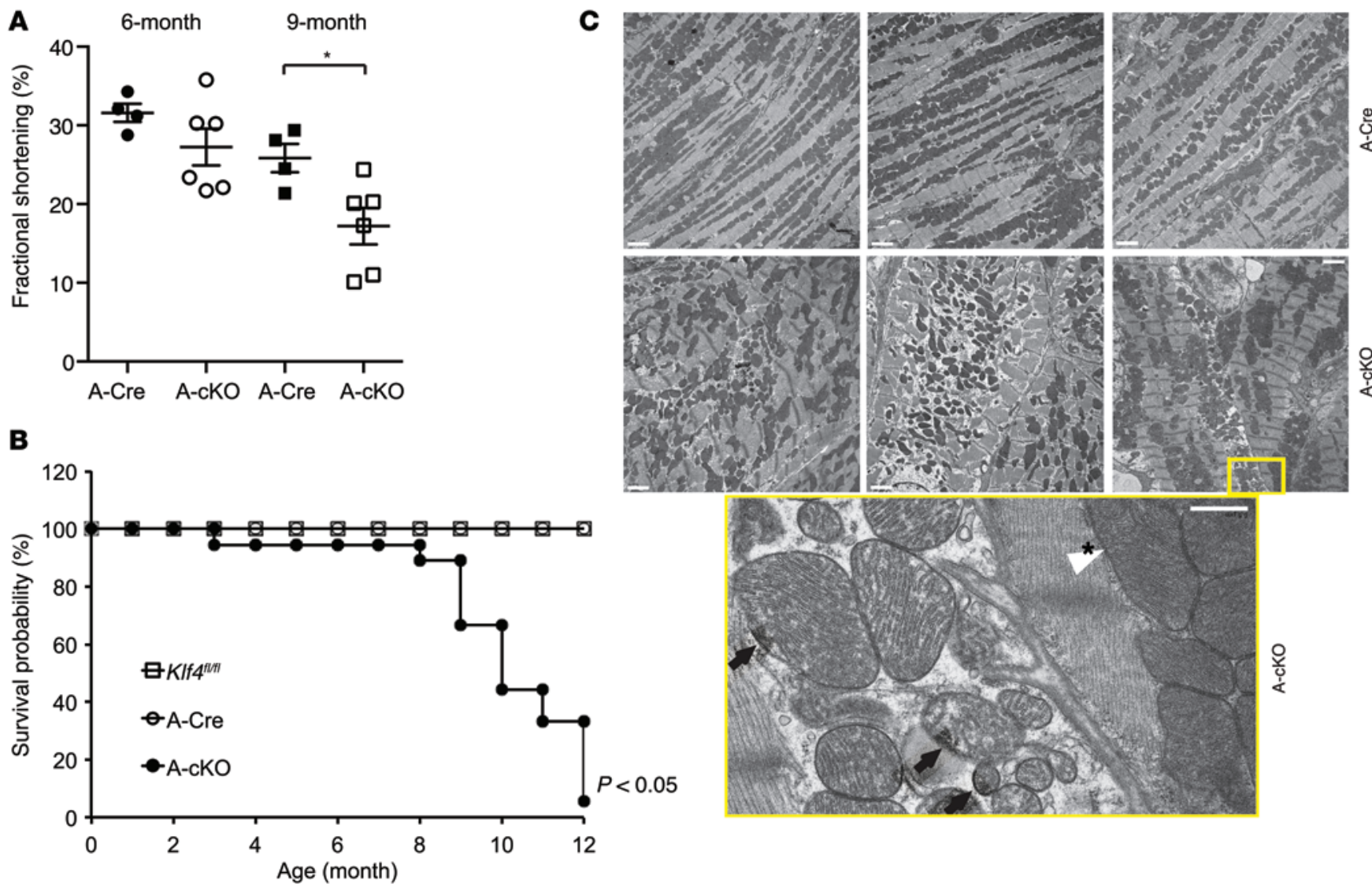

$\frac{0}{\dot{y}}$

Figure 3. Cardiac dysfunction and accumulation of injured mitochondria in aged KLF4-deficient heart. (A) Cardiac function declined with age in A-cKO mice. $n=4 .{ }^{*} P<0.05$, Student's $t$ test with Bonferroni correction. (B) Shortened life span of A-cKO mice. $n=18$ in A-cKO group, $n=20$ in each control

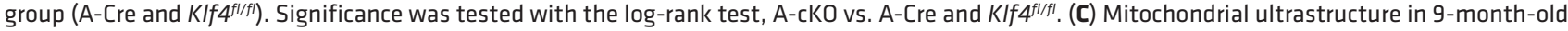
mouse heart. Representative images are from 3 pairs of mice. The boxed area is shown at high magnification. In the high-magnification image, the white arrowhead indicates healthy mitochondria, and black arrows indicate damaged mitochondria with degeneration (pale) and fragmentation (small, round). Scale bar: $2 \mu \mathrm{m}$ (low-magnification images); $0.5 \mu \mathrm{m}$ (high-magnification images).

ventricular myocytes (NRVMs) with acute KLF4 silencing (Figure 2D), and, conversely, transient overexpression of KLF4 enhanced mitochondrial respiration in NRVMs (Figure 2E), strongly indicating a cell-intrinsic role of KLF4 on mitochondrial function. Collectively, these results support a key role for KLF4 in mitochondrial OXPHOS function and energetics in the myocardium.

KLF4 deficiency impairs myocardial function with age. The finding that young A-cKO mice exhibited normal cardiac function under basal conditions was somewhat surprising in light of the basal defects noted in isolated mitochondrial respiration studies. We hypothesized that this basal defect may become more apparent and affect cardiac function over time (i.e., in aged mice), as it had in response to an acute stress like TAC. We performed serial echocardiography on a cohort of animals over a 9- to 12-month period. A small reduction in fractional shortening was noted at 6 months, and this progressed to a significant reduction by 9 months of age (Figure 3A). These animals also exhibited reduced survival, with nearly $100 \%$ mortality by 1 year of age (Figure 3B). Importantly, like the hearts after TAC, aged KLF4-deficient hearts displayed increased mitochondrial fragmentation, disarray, and degeneration, indicating enhanced mitochondrial injury over time (Figure 3C and Supplemental Figure 4). Collectively, these data suggest that the mitochondrial defects resulting from KLF4 defi- ciency are significant and manifest in response to pathological (pressure overload) or physiological stress (aging).

KLF4 is essential to postnatal mitochondrial biogenesis and dynamics in the heart. Since genes that regulate mitochondrial biogenesis and dynamics were altered in KLF4-deficient myocardium (Figure 1E), we next sought to determine whether KLF4 also regulates mitochondrial biogenesis and dynamics in the heart. It has been reported that these processes are quite rare in the adult myocardium but critical for cardiac adaptation in the postnatal period $(5,14,25)$. Thus, we chose to study perinatal cardiac development, as this period is notable for robust changes in mitochondrial biogenesis and dynamics.

As a first step, we studied myocardial KLF4 expression during the perinatal period and found that expression of KLF4 was significantly induced after birth in both human and rodent hearts, in parallel with PPARGC1A (Figure 4A), which encodes PGC-1 $\alpha$, a transcriptional coactivator that regulates mitochondrial biogenesis and dynamics $(5,14,26)$. Since the Myh6-Cre we used only achieves $70 \%-80 \%$ deletion of floxed gene allele by 2 weeks of age, making it unsuitable for the study of perinatal cardiac development (27), we chose a Cre line driven by a 2.8-kb Tagln promoter (also known as Sm22) that efficiently deletes floxed gene alleles in 
A Human heart

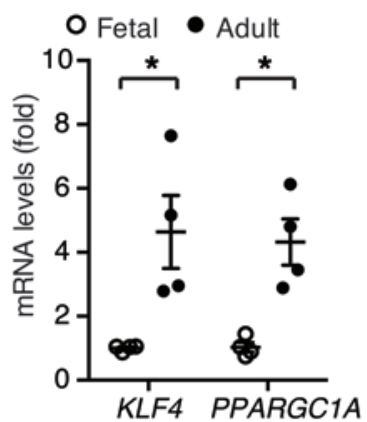

C

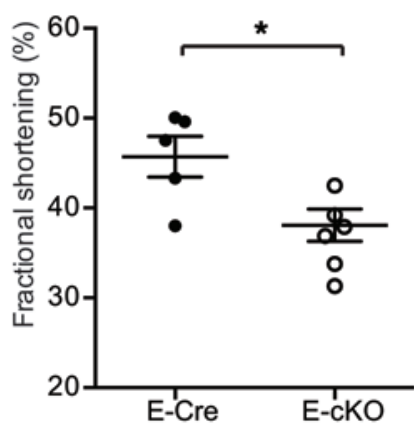

Mouse heart



D

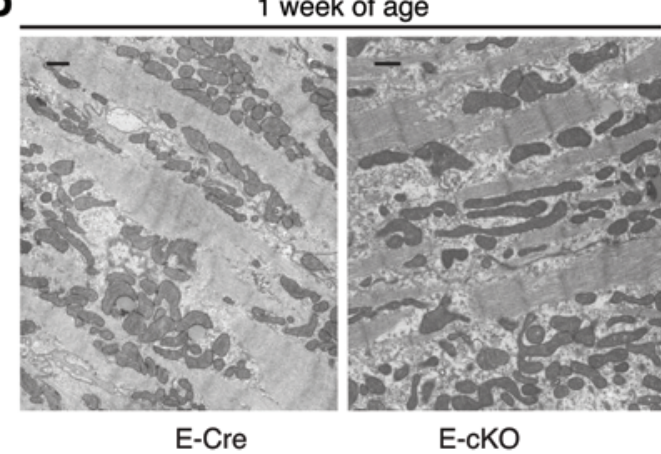

E-Cre

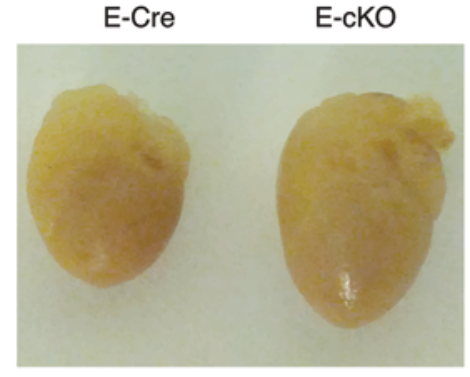

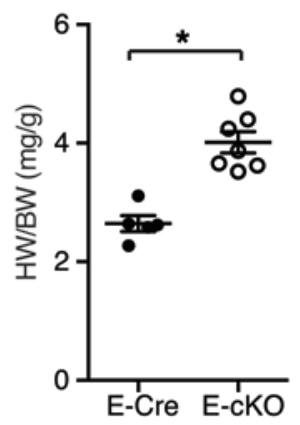

2 weeks of age

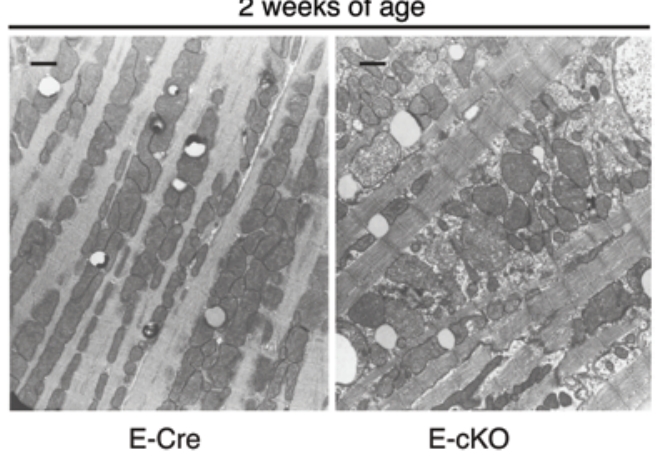

G Heart
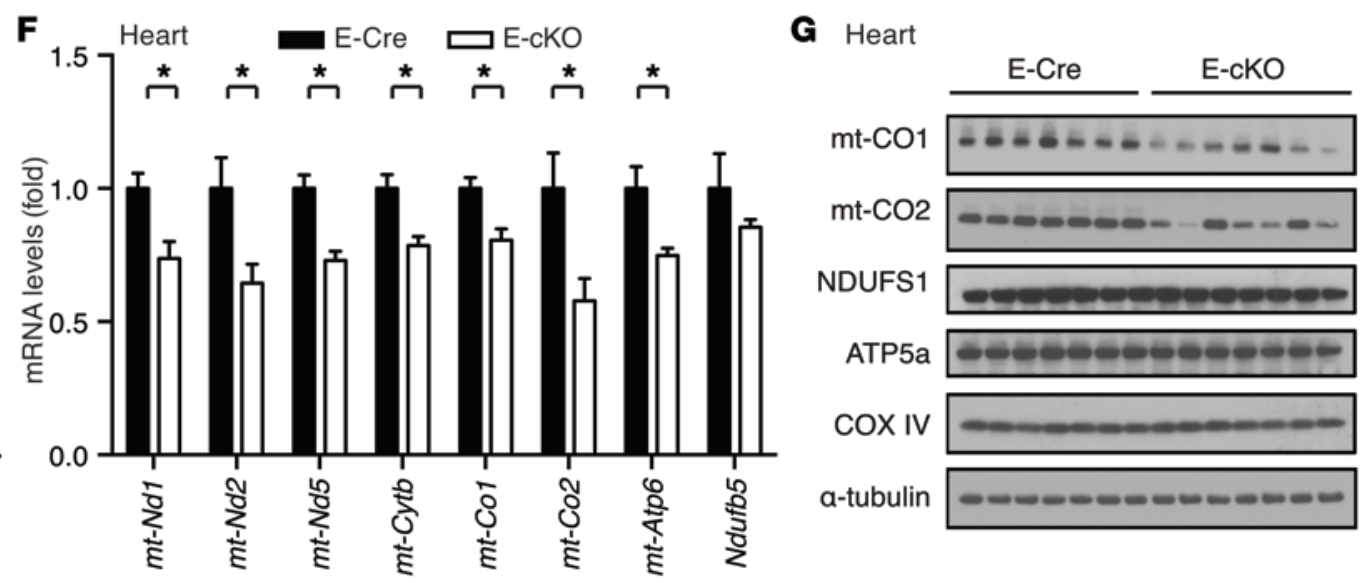

H
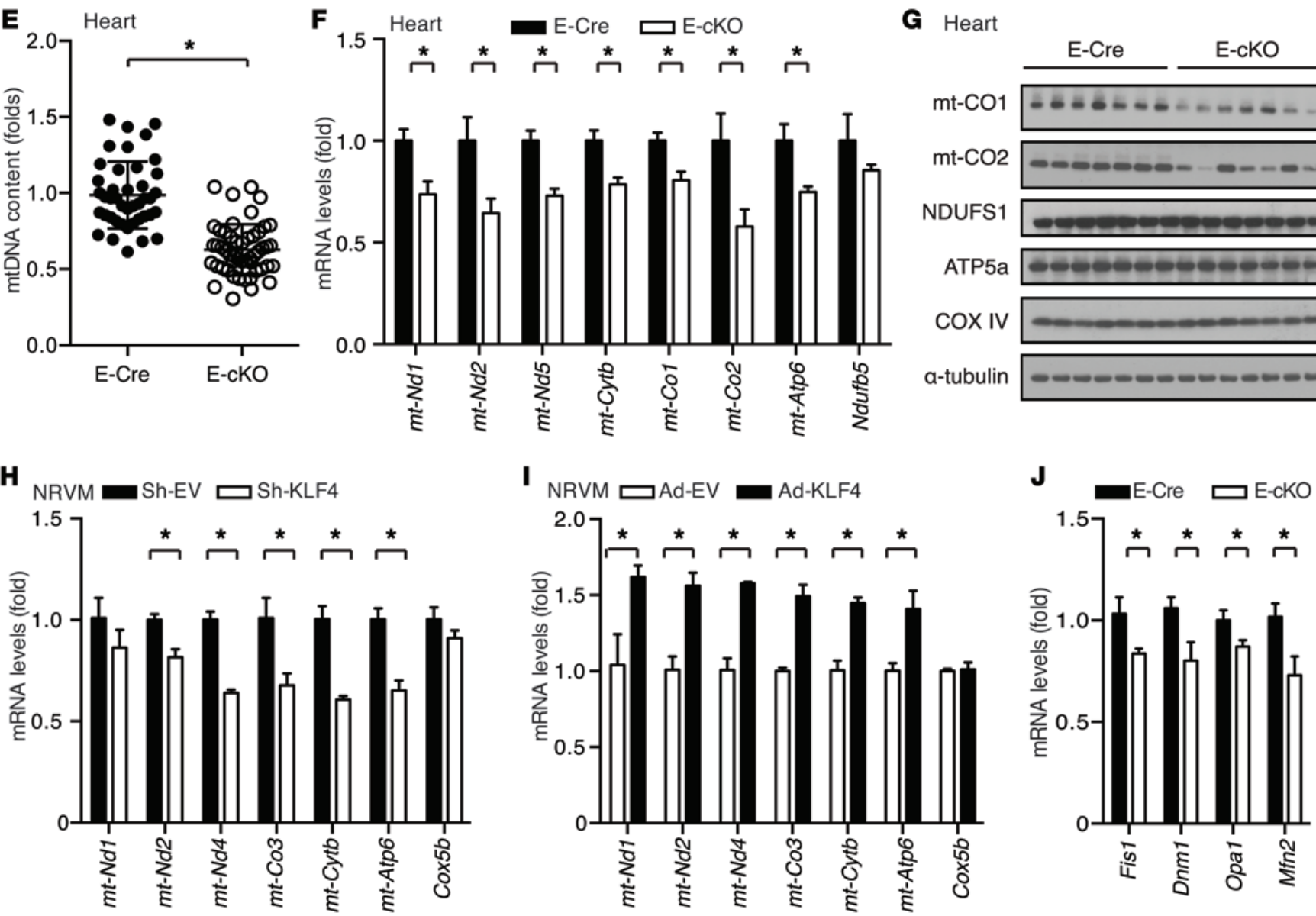
Figure 4. Myocardial KLF4 is required for postnatal mitochondrial biogenesis and cardiac maturation. (A) Expression of KLF4 and PPARGC1A in human and mouse hearts. Prenatal data were used as controls. $n=4$ for each data point. (B) Heart size and weight. (C) Heart contractility, as assessed by echocardiography. (B and C) $n=5-7$ at 4 weeks of age. (D) Myocardium ultrastructure. Representative images are shown $(n=3$ in each group). Scale bar: $1 \mu \mathrm{m}$. (E) Cardiac mitochondrial genomic DNA, as assessed by qPCR. Mitochondrial genome copies (mtDNA) were normalized to nuclear DNA content. $n=10$ in each genotype. Dots showed qPCR results from 5 pairs of primers ( $m t-N d 1, m t-N d 4, m t-C 01, m t-C_{02}$, and mt-Atp6). (F) Expression of genes encoded by the mitochondrial genome. Ndufb5, a nuclear-encoded gene, was included as control. (C) Protein levels of mitochondrial- and nuclear-encoded genes. Each lane represents one animal. (H) Acute knockdown or (I) overexpression of KLF4 in NRVMs affected expression of mitochondrial-encoded genes. Cox5b, a nuclear-encoded gene, was included as control. (J) Deficiency of cardiac KLF4 impaired the expression of genes that regulate mitochondrial dynamics. Heart samples: $n=10$. NRVM samples: $n=3$. DNA/RNA/protein analysis was performed with 2-week-old animals. ${ }^{*} P<0.05$, Student's $t$ test with Bonferroni correction.

the heart at E9.5 (28) (Tagln-Cre, hereafter designated E-Cre) to generate myocardial KLF4 deficiency in mice during the critical postnatal development period (Tagln-Cre Klf $4^{f / f l}$ mice, hereafter designated E-cKO mice) for mitochondrial biogenesis.

Consistent with previous observations (29), E-cKO mice exhibited approximately $50 \%$ mortality within 2 weeks after birth (data not shown). Strikingly, surviving E-cKO animals displayed severe cardiac dysfunction characterized by cardiac hypertrophy and LV dilation along with reduced contractile function by 4 weeks of age (Figure 4, B and C). Although the Cre we used is known to be expressed in smooth muscle cells (28), no effect was observed on myocardial vessel density, fibrosis, or the expression of hypoxia-inducible genes, suggesting that vascular function was intact (Supplemental Figure 5, A-C). In addition, it has been shown that KLF4 is not expressed in smooth muscle cells during development (29). However, we observed a significant reduction of a set of metabolic and mitochondrial genes, similar to that observed in adult KLF4 deficiency (Supplemental Figure 5D). Thus, we hypothesized that the cardiac phenotype and premature death observed in the E-cKO animals occurs as a result of cardiac maladaptation to postnatal developmental stress.

Electron microscopic evaluation of LV tissue from E-cKO mice revealed reduced myocardial mitochondrial volume density, with mitochondrial disarray, degeneration, fragmentation, elongation, and increased heterogeneity: differences appreciable at 1 week and more pronounced at 2 weeks (Figure 4D and Supplemental Figure 6). Consistent with the observed reduction in mitochondrial density, an approximately $30 \%$ reduction in mitochondrial genomic DNA content was confirmed by qPCR (Figure 4E) and multiple mitochondrial-encoded genes were also reduced at both the mRNA and protein levels (Figure 4, F and G). The correlation between KLF4 levels and expression of mitochondrial-encoded genes was recapitulated in NRVMs with acute KLF4 silencing and overexpression, indicating a cell-autonomous effect (Figure 4, $\mathrm{H}$ and I). We also observed a KLF4 dependency on a number of target genes involved in mitochondrial dynamics (Figure 4J), a finding consistent with the presence of both small (fragmented) and long (giant) mitochondria in E-cKO hearts (Figure 4D and Supplemen- tal Figure 6). Consistent with the above-mentioned mitochondrial abnormality, myocardial glycogen content was slightly increased in the E-cKO group (Supplemental Figure 7). Collectively, these results support a critical role for KLF4 in postnatal cardiac mitochondrial biogenesis, dynamics, and maturation.

KLF4 is requisite for the ERR/PGC-1 transcription module. The transcriptional regulation of cardiac metabolism and mitochondrial function has resided largely in the domain of aforementioned nuclear receptors. Intriguingly, we noted that the cardiac phenotypes of KLF4-deficient mice in response to physiologic (postnatal adaptation) and pathologic (pressure overload) stress were strikingly similar to those of Ppargc1a, Ppargc1b, and Esrra single or compound KO mice with respect to cardiac mitochondrial dysfunction, impaired postnatal mitochondrial biogenesis, and dynamics $(5,11,13,14,30)$. Thus, we hypothesized that KLF4 may interact with the ERR/PGC-1 signaling pathway.

We studied cytochrome $c$ (Cycs), PPAR $\alpha$ (Ppara), and pyruvate dehydrogenase kinase, isozyme $4(P d k 4)$ as classic direct targets of the ERR/PGC-1 module (12, 31, 32). Promoter analyses revealed multiple consensus KLF-binding elements (CA/GCCC, designated KRE for KLF response element) in proximity to a nuclear receptor response element (NRRE) (Figure 5A and Supplemental Figure 8). ChIP and promoter reporter studies confirmed that KLF4 directly binds to and induces all 3 promoters (Figure 5A and Supplemental Figure 8, A and B). Cotransfection of KLF4 with ERR/PGC-1 resulted in a synergistic induction of target promoters (Figure 5B and Supplemental Figure 8, C and D). Conversely, knockdown of KLF4 attenuated ERR/PGC-1-mediated promoter transactivation (Figure 5C). Next, we sought to determine whether direct binding of $\mathrm{KLF} 4$ onto $\mathrm{KRE}$ is required for promoter transactivation. As there are $4 \mathrm{KRE}$ sites and $1 \mathrm{NRRE}$ site on the 1,766-bp mouse Cycs promoter used in this study, we first performed 5 '-end serial truncation to locate the crucial KRE sites and found that the transactivation synergy was significantly attenuated when truncation included the NRRE vicinity (Figure 5D). Further promoter sequence alignment analysis indicates that $2 \mathrm{KRE}$ sites flanking the NRRE are conserved from rodent to human, and mutating either or both of the 2 conserved KRE sites significantly attenuated the transactivation synergy between KLF4 and the ERR/ PGC-1 module, suggesting that direct KLF4 binding onto the promoter is required for optimal transcription (Figure 5E). Similar promoter truncation analysis with the Ppara promoter demonstrated that a cluster of $9 \mathrm{KRE}$ sites residing between the 2 NRRE sites is required for KLF4-ERR/PGC-1 synergy (Supplemental Figure 8E); while the NRRE site of the $P k d 4$ promoter appeared to mediate most promoter function (Supplemental Figure $8 \mathrm{~F}$ ). Given that Ppara is a major regulator of cardiac FAO, we examined KLF4 effects on FAO in NRVMs. KLF4 overexpression increased expression of FAO genes and FAO rates, while knockdown blunted induction mediated by WY16463, a synthetic ligand for PPAR $\alpha$ (Supplemental Figure 9). In contrast, KLF4 had minimal effects on glycolysis but affected glucose oxidation, likely through regulation of mitochondria function (Supplemental Figure 10).

We next sought to determine the molecular basis for the observed transcriptional synergy. Coimmunoprecipitation assays demonstrated that KLF4 interacts with $\operatorname{ERR} \alpha$ and, to a lesser degree, with PGC- $1 \alpha$ and interacts minimally with PGC-1 $\beta$ (Fig- 
A

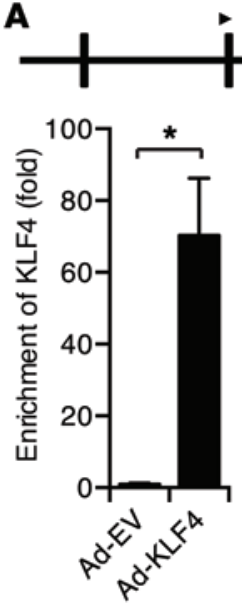

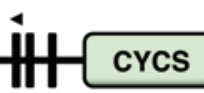

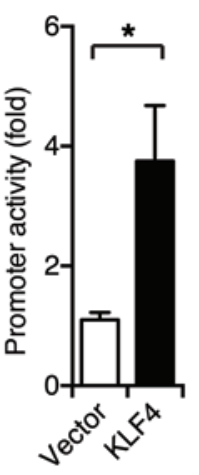

B

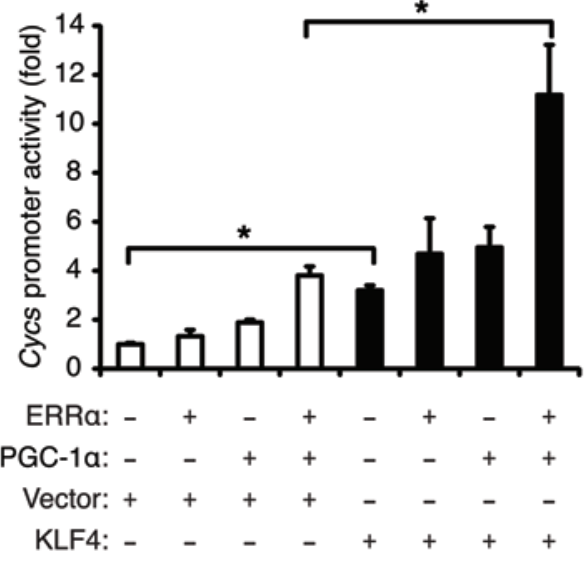

C

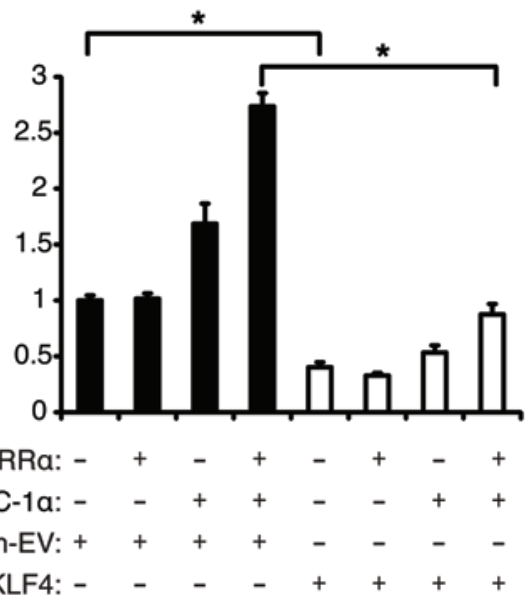

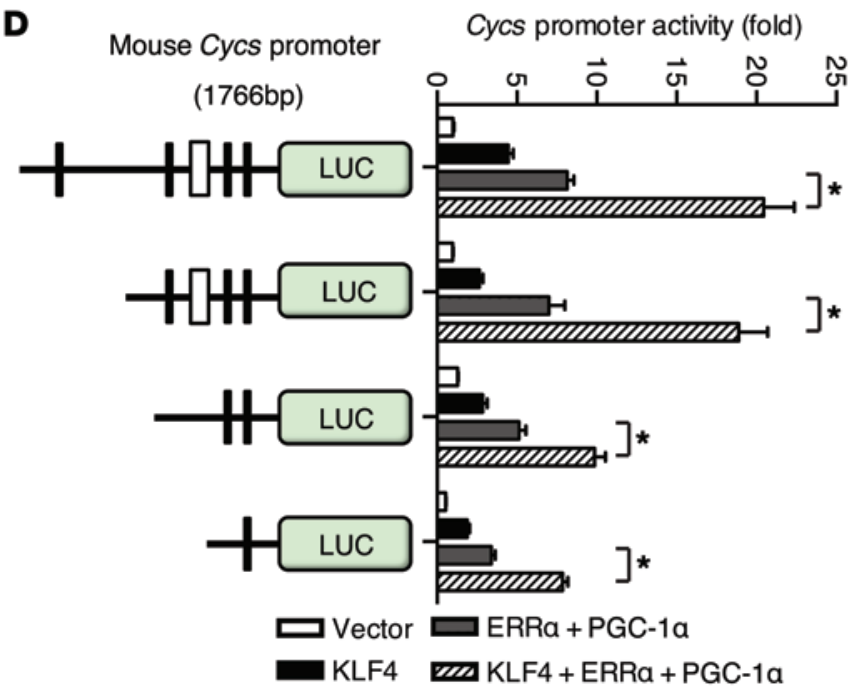

D

$\mathbf{F}$

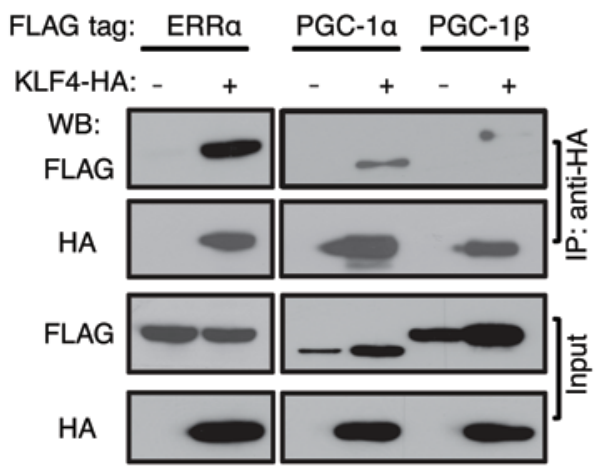

E Conserved:

KRE1 KRE2


WT : $\mathrm{CACCC}$ mutant : CAGG \

Cycs promoter activity (fold)

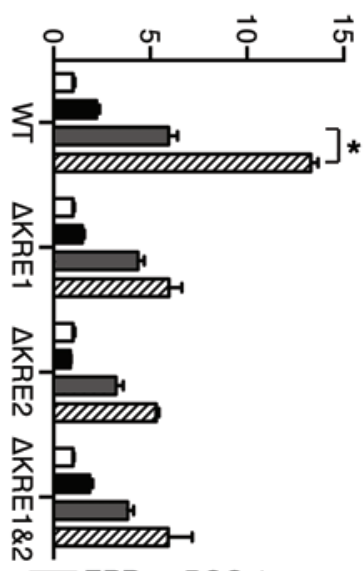

ERRa + PGC-1a

m KLF4 + ERRa + PGC-1a

\section{G}

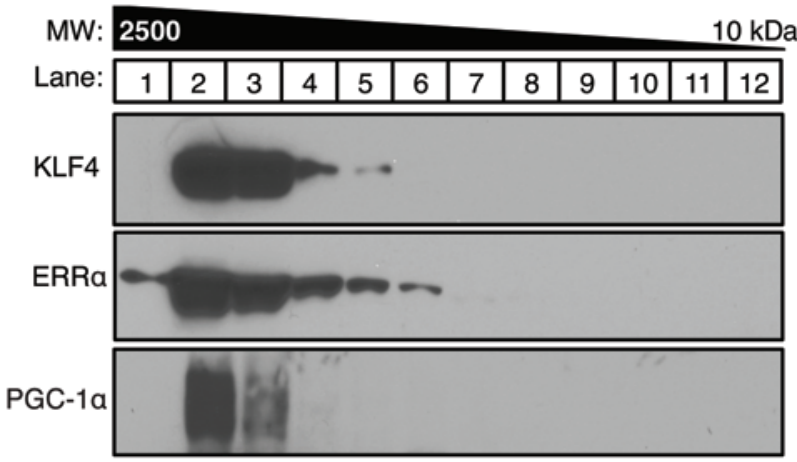

H

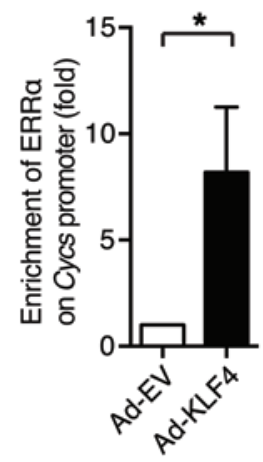

Figure 5. KLF4 binds to and cooperates with the ERR/PGC-1 transcriptional module in regulation of target gene transcription. (A) Recruitment of KLF4 onto endogenous Cycs promoters and activation of the promoter reporter. Black rectangles indicate KRE and the white rectangle indicates NRRE. Arrowheads indicate PCR regions in ChIP assays. (B and C) Synergistic cooperativity between KLF4 and the ERR/PGC-1 complex on target promoters. (D) Promoter truncation analysis. (E) Site mutation in Cycs promoter. (F) Protein-protein interaction between KLF4 and PGC-1 $\alpha /$ PGC-1B/ERR $\alpha$. (C) Gel filtration chromatography analysis. (H) KLF4 augmented the recruitment of ERR $\alpha$ to endogenous Cycs promoter in NRVMs. $n=3 .{ }^{*} P<0.05$, Student's $t$ test with Bonferroni correction.

ure 5F). Interactions between KLF4 and ERR $\alpha$, PGC- $1 \alpha$ and KLF4, and ERR $\alpha$ and PGC- $1 \alpha$ further suggest the existence of a KLF4/ ERR/PGC-1 trimolecular complex. To test this idea, we performed gel filtration chromatography and found that all 3 molecules were eluted in the same fractions, supporting the existence of a trimo- lecular complex (Figure 5G). Finally, KLF4 augmented the recruitment of ERR $\alpha$ to the endogenous Cycs promoter (Figure $5 \mathrm{H}$ ).

To gain further mechanistic insight, we mapped the protein domains that mediate the KLF4-ERR $\alpha$ interaction by coimmunoprecipitation assays using a series of truncated KLF4 and ERR $\alpha$ 
A

KLF4(1-479)

KLF4(1-397)

KLF4(1-181)

KLF4(118-479)

KLF4(118-397)

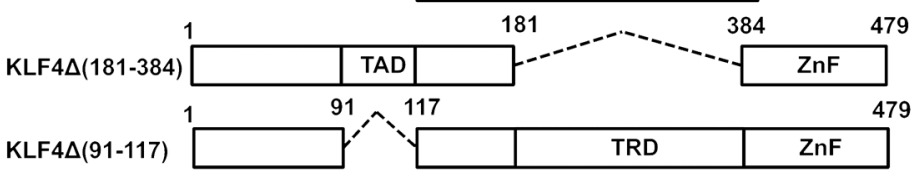

B

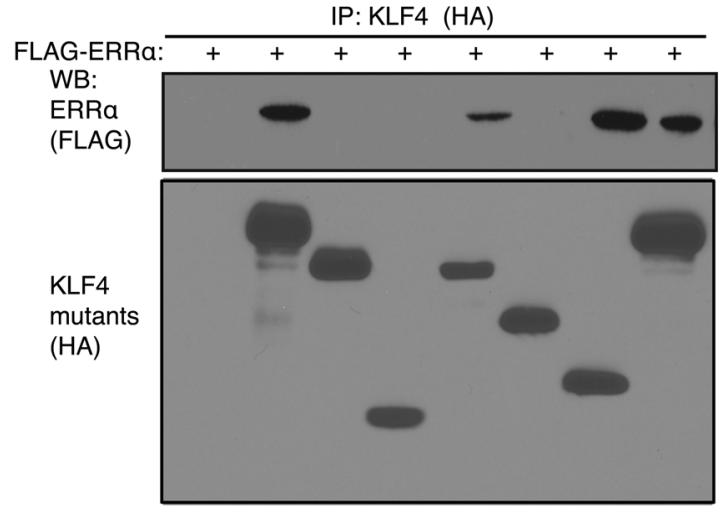

KLF4:

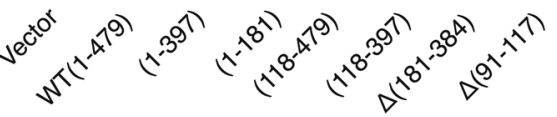

C

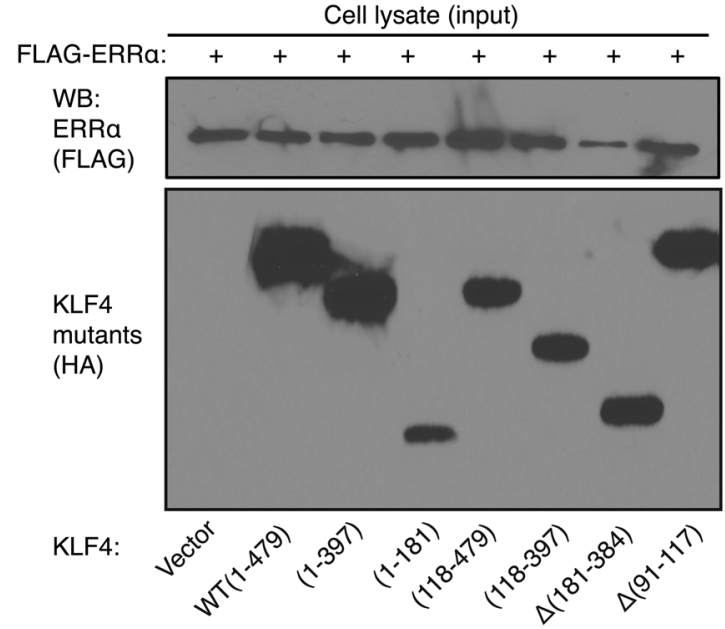

D $\operatorname{ERRa(1-422)}$

\begin{tabular}{|c|c|c|}
\hline & & 403 \\
\hline & DBD & LBD \\
\hline \multicolumn{3}{|c|}{1} \\
\hline & DBD & LBD \\
\hline \multicolumn{3}{|c|}{1} \\
\hline & DBD & \\
\hline
\end{tabular}

E HA-KLF4: $\frac{\text { IP: ERRa (FLAG) }}{+++++} \frac{\text { Cell lysate (input) }}{+++++}$ KLF4 (HA)

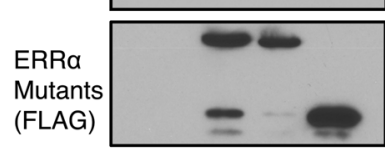

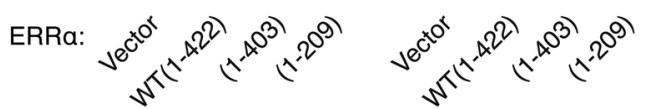

$\mathbf{F}$

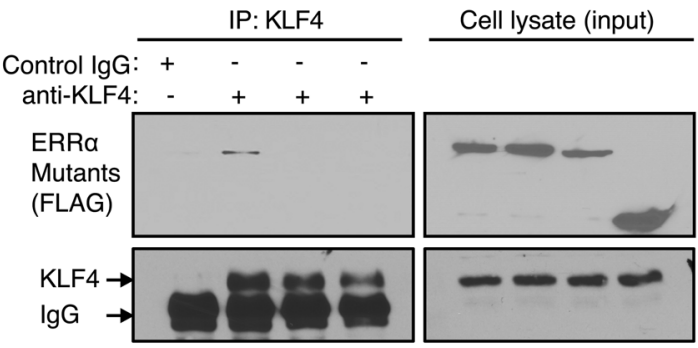

ERRa:
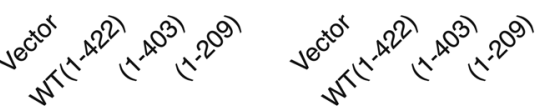

G

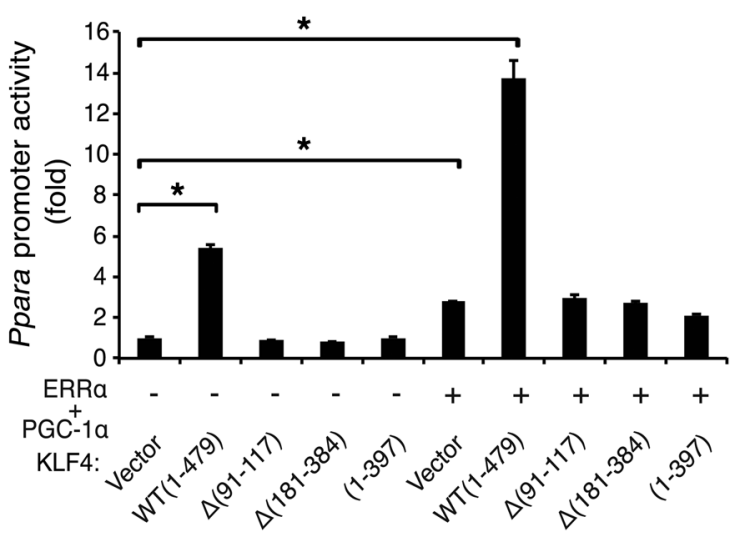

Figure 6. Interaction and cooperativity between KLF4 and the ERR/PGC-1 module. (A) Full-length and truncated KLF4 proteins. TAD, transactivation domain; TRD, transrepression domain. (B and C) Protein-protein interaction between full-length ERR $\alpha$ (FLAG-tagged) and different forms of truncated KLF4 (HA-tagged). (D) Full-length and truncated ERR $\alpha$ proteins. DBD, DNA-binding domain; LBD, ligand-binding domain. (E and F) Protein-protein interaction between full-length KLF4 (HA-tagged and endogenous KLF4) and different forms of truncated ERR $\alpha$ (FLAG-tagged). (C) Functional cooperativity between the ERR $\alpha /$ PGC-1 $\alpha$ complex and different forms of truncated KLF4. $n=3$. ${ }^{*} P<0.05$, Student's $t$ test with Bonferroni correction.

proteins. This study showed that the zinc finger domain $(\mathrm{ZnF})$ of KLF4 mediates the interaction with ERR $\alpha$, as all KLF4 mutants that carry the $\mathrm{ZnF}$ domain (aa 384-479), but not the $\mathrm{ZnF}$ deletion mutants, were able to pull down ERR $\alpha$ (Figure 6, A-C). In addition, the activation function-2 homology region (AF-2) of ERR $\alpha$, which is also critical for interacting with PGC-1 $\alpha$ (33), is required for its interaction with KLF4 (Figure 6, D-F). Of note, although the $\mathrm{ZnF}$ domain of KLF4 is sufficient to mediate its protein-protein interaction with ERR $\alpha$, transcriptional activity of the KLF4/ ERR $\alpha /$ PGC-1 $\alpha$ complex requires the full-length KLF4 (Figure 6G). 

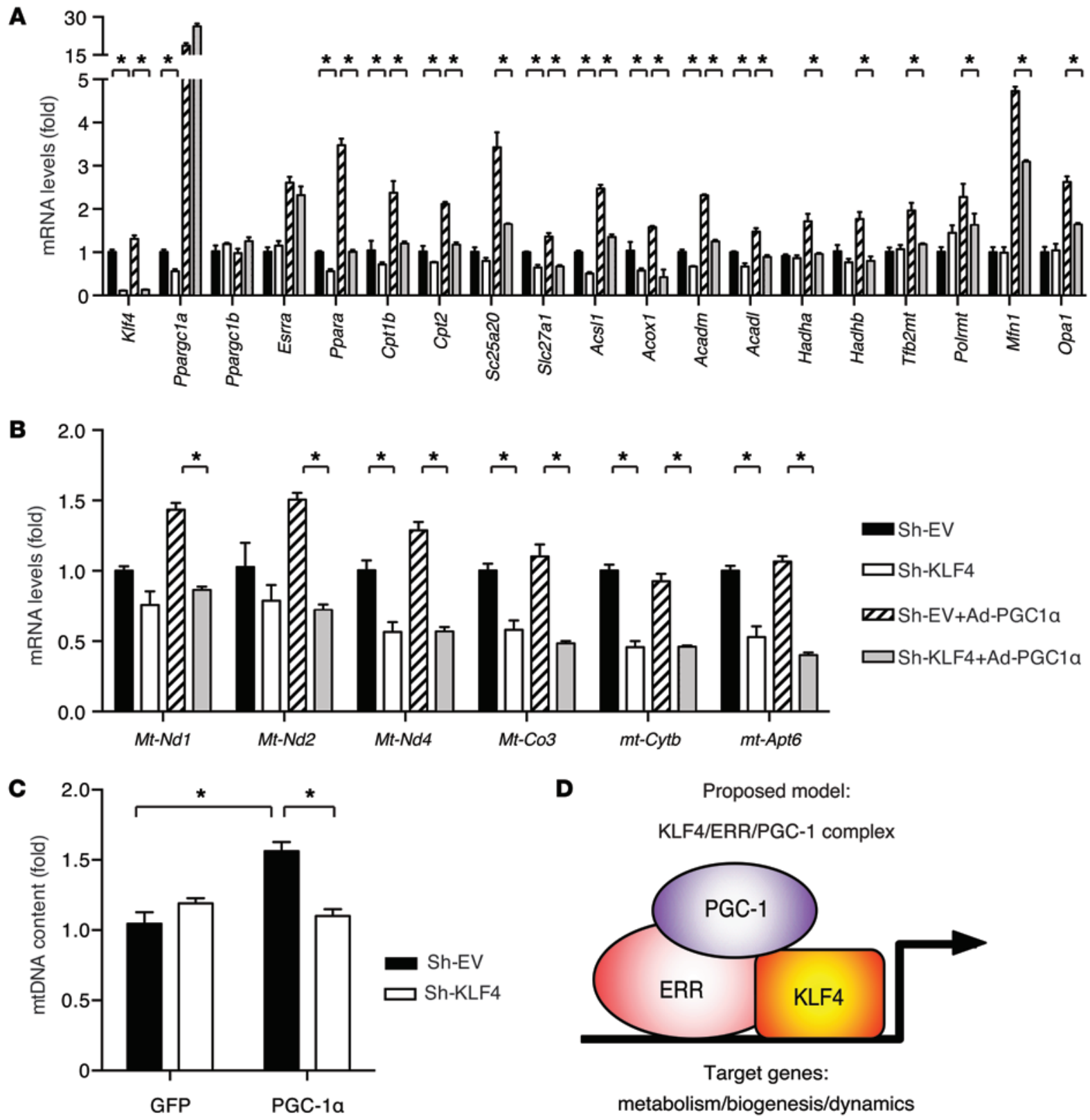

Figure 7. KLF4 is requisite for optimal transcriptional function of the ERR/PGC-1 complex. (A and B) KLF4 is requisite for PGC-1 $\alpha$-mediated induction of (A) metabolic and (B) mitochondrial genes in NRVMs. (C) KLF4 deficiency impaired PGC-1 $\alpha$-mediated mitochondrial biogenesis in NRVMs. $n=3$. ${ }^{*} P<0.05$, Student's $t$ test with Bonferroni correction. (D) Proposed mechanistic model of the KLF4/ERR/PGC-1 transcriptional complex.

To determine whether KLF4 is requisite for ERR/PGC-1 signaling, we examined PGC-1 $\alpha$-induced target gene expression in NRVMs in the presence or absence of KLF4. As expected (14, 34), numerous genes involved in mitochondrial FAO, OXPHOS, biogenesis, and dynamics were induced by acute overexpression of PGC-1 $\alpha$ in NRVMs. However, such induction was either significantly attenuated or completely abolished when KLF4 was silenced (Figure 7, A and B). We also noted that KLF4 knockdown had effects on specific mitochondrial-encoded genes that are independent of PGC-1 $\alpha$ (Figure 7B), suggesting the possibility that KLF4 may regulate mitochondrial genome transcription. Finally, KLF4 silencing significantly blunted the PGC- $1 \alpha$-induced mitochondrial biogenic response (26), suggesting that KLF4 is requisite for the mitochondrial biogenesis machinery (Figure 7C). Collectively, these data demonstrate that KLF4 binds to, cooperates with, and is requisite for optimal transcriptional function of the ERR/PGC-1 module, likely through the formation of a KLF4/ ERR/PGC-1 trimolecular complex (Figure 7D).

KLF4 regulates autophagy in cardiomyocytes. The above results indicate that, in response to physiologic and pathologic stress, mitochondrial homeostasis is dependent upon KLF4 expression. The accumulation of the fragmented/damaged mitochondria in the KLF4-deficient state raises the question whether loss of KLF4 may also affect the process of autophagic clearance.

To address this, we first examined the conversion of LC3-I to LC3-II, a hallmark of autophagy induction, in TAC-stressed A-cKO 
hearts and found that KLF4 deficiency reduced LC3-II accumulation (Figure 8A). Further, in vitro KLF4 gain- and loss-of-function studies, in the setting of autophagy induced by a mitochondrial uncoupling reagent (carbonyl cyanide $\mathrm{m}$-chlorophenylhydrazone [CCCP]), recapitulated the in vivo KLF4-dependent effects on LC3-II accumulation (Figure 8A). To monitor autophagy flux, we used bafilomycin A1 (BFA) to block lysosomal removal of engulfed LC3-II and confirmed that KLF4 promotes autophagy flux (Figure $8 \mathrm{~A}$ ). As previously reported, mitochondria that escape from autophagy could trigger inflammatory responses in the myocardium leading to cardiac dysfunction (35). The impaired autophagy seen in KLF4-deficient hearts may not only jeopardize energy production but also induce myocardial inflammation, as revealed by the inflammatory signature from microarray analysis (Figure $1 \mathrm{~B})$, and Il6 induction in TAC hearts in vivo and in CCCP-treated NRVMs in vitro (Supplemental Figure 11). Thus, KLF4 deficiency appears to impair autophagy flux, leading to accumulation of damaged mitochondria and inflammation in the myocardium.

PINK1/parkin pathway plays a major role in mitochondrial clearance. Depolarized mitochondria are recognized by PINK1 and labeled through parkin-mediated ubiquitination, which subsequently activates autophagy-related genes (ATGs) to engage autophagy process (36). Through a cascade of protein interaction and modification, ATGs form autophagosomes to engulf damaged mitochondria and deliver them to lysosomes for degradation (37). We assessed the activation of PINK1/parkin signaling in myocardium following TAC. KLF4 deficiency did not significantly affect the mitochondrial enrichment of PINK1 and parkin, subsequent ubiquitination of mitochondrial proteins, or the recruitment of p62, suggesting intact PINK1/parkin signaling (Figure 8B). Unc-51-like kinases 1 and 2 (Ulk1 and Ulk2), the mammalian homologs of Atg1, are essential in the initiation of autophagosome formation and, thus, serve as a central regulator of autophagy (38-40). It has been shown that ULK1 is activated through phosphorylation at the Ser555 residue by adenosine monophosphate-activated protein kinase (AMPK). We examined myocardial ULK1 signaling and found that TAC-induced ULK1 phosphorylation was markedly attenuated in KLF4-deficient hearts (Figure 8C). These data were recapitulated in NRVMs with acute KLF4 overexpression or knockdown (Figure 8C). Further, acute silencing of Ulk1 markedly attenuated KLF4-induced LC3-II conversion in NRVMs, indicating an indispensable role of ULK1 in KLF4-mediated autophagy (Figure 8D).

We next sought to understand how KLF4 affects ULK1 activation. Given that the phosphorylation of AMPK was not affected by KLF4 levels in NRVMs in vitro and actually increased in KLF4-deficient myocardium in vivo (Figure 2C and Figure $8 C$ ), the KLF4-mediated alterations in ULK1 phosphorylation were unlikely to have resulted from changes in AMPK activity. Indeed, the phosphorylation of acetyl-CoA carboxylase, a direct target of AMPK, was not affected by KLF4 in NRVMs (Supplemental Figure 12). However, we noted that ULK1 total protein levels paralleled ULK1 phosphorylation levels and were positively correlated to KLF4 levels (Figure 8C), suggesting a possibility of KLF4-mediated transcriptional regulation. Consistent with this hypothesis, KLF4-mediated transcription of Ulk1 was confirmed by qPCR in the heart and NRVMs (Figure 1C and Figure 8E). To determine whether Ulk1 is a direct transcriptional target of KLF4, we cloned a 1,071-bp promoter region upstream of the transcription start site of the rat Ulk1 gene and found it was inducible by KLF4 in NRVMs (Supplemental Figure 13A). Notably, KLF4 appeared to directly transactivate the Ulk1 promoter, as a KLF4 mutant lacking the ZnF DNA-binding domain had no effect on promoter induction, and this effect was specific to KLF4, as a closely related family member - KLF2 - failed to induce the Ulk1 promoter (Supplemental Figure 13B). Promoter truncation studies revealed that multiple KRE sites in the proximal region of the transcription start site were crucial to KLF4-mediated transcription (Figure 8F). Subsequent ChIP studies confirmed binding of KLF4 at this region of endogenous $U l k 1$ promoters in NRVMs, suggesting that Ulk1 is likely a direct target of KLF4 (Figure 8G). Similar KLF4-mediated regulation and dependency of Ulk2 was observed as well (Supplemental Figure 14). Collectively, these data demonstrated that KLF4 mediates autophagy flux through transcriptional regulation of $U l k 1 / 2$.

Finally, to gain a comprehensive understanding of KLF4-mediated transcriptional regulation of autophagy, we screened the entire autophagy pathway using a commercially available qPCR array and found that a broad spectrum of autophagy genes was differentially regulated by KLF4 overexpression and deficiency in NRVMs (Figure $8 \mathrm{H}$ and Supplemental Figure 15A). We further confirmed that 31 of the 54 genes that had reduced expression after KLF 4 knockdown were reduced in KLF4-deficient hearts in vivo, which recapitulated the in vitro finding (Figure 8I and Supplemental Figure 15B). These data suggest that KLF4 may transcriptionally regulate autophagy at multiple steps, making it a novel and central transcriptional regulator of this process. Thus, through the direct transcriptional regulation of the autophagy pathway, KLF4 may affect mitochondrial clearance, the last phase of mitochondrial life cycle.

\section{Discussion}

Mitochondria serve as the power plant for cardiomyocytes, providing their energetic needs in the form of ATP to sustain contractile function. Mitochondrial OXPHOS function is under tight control to meet cellular energetic demand. Further, mitochondria undergo biogenesis, fission, and fusion to maintain homeostasis. In this study, we demonstrate that KLF4 regulates mitochondrial OXPHOS, biogenesis, and dynamics in the heart, likely through cooperation with the ERR/PGC-1 pathway. Moreover, KLF4 also directly regulates autophagy genes. These findings position KLF4 as a critical component of the transcriptional circuitry regulating cardiac mitochondrial homeostasis (summarized in Figure 9).

To date, the transcriptional regulation of cardiac energy metabolism and mitochondrial function has largely been attributed to the ERR/PGC-1 transcriptional module, which plays an integrative role in relaying developmental and physiological signals to a key subset of transcription factors to direct the coordinate expression of nuclear and mitochondrial genes (16). We believe that our data demonstrate that KLF4 is a novel and essential component of this transcriptional module, and the basis for this conclusion is manifold. First, KLF4 deficiency in both mature and developing hearts shows striking parallels to the mitochondrial phenotype observed in the Ppargcla Ppargc1b KO (single and compound KO) and Esrra $\mathrm{KO}$ animals, including altered cellular metabolism, mitochondrial biogenesis, dynamics, and cardiac dysfunction. Second, KLF4 depletion attenuates classic cellular actions of PGC-1, including 
A
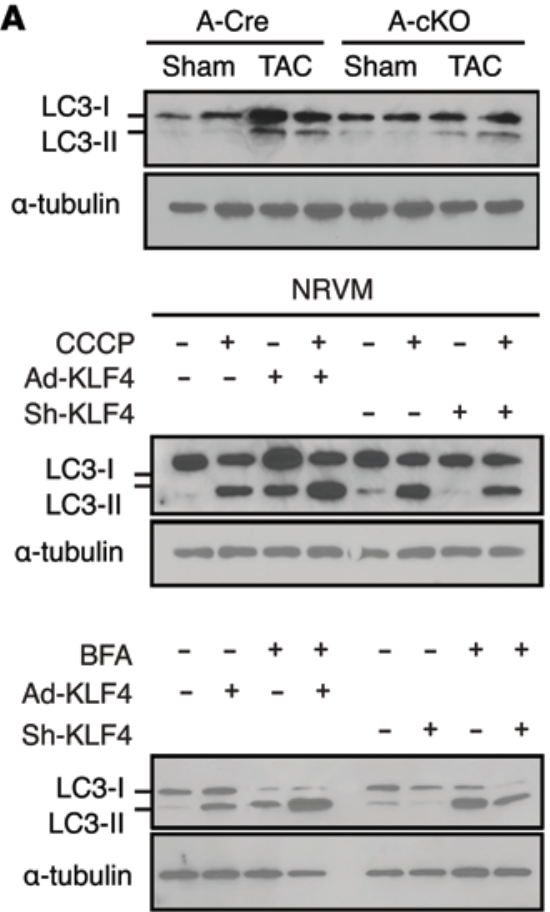

D

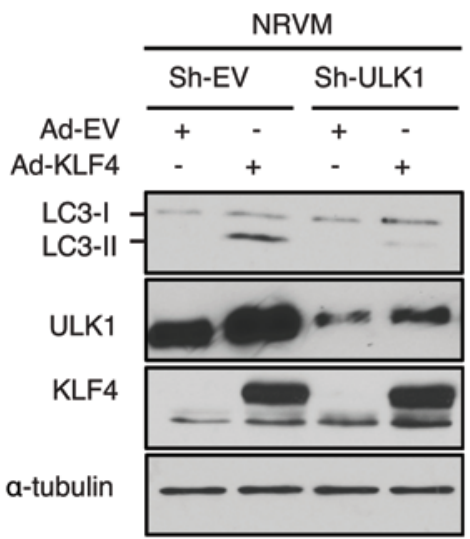

G

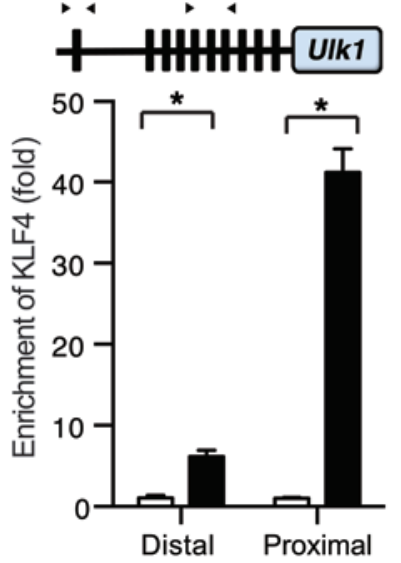

B

$\frac{\text { Mitochondria }}{\frac{\text { Sham }}{\text { Cre KO }} \frac{\text { TAC }}{\text { Cre KO }}}$

PINK1

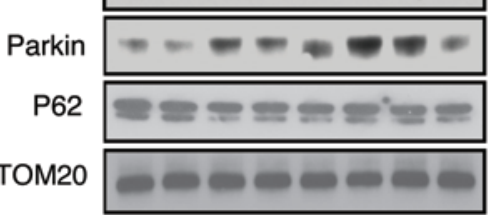

Mitochondria

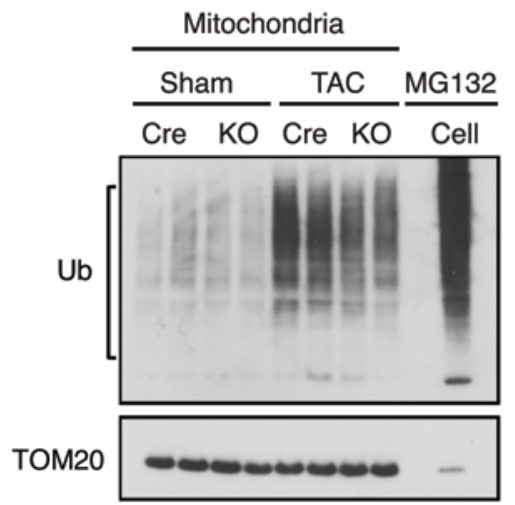

C
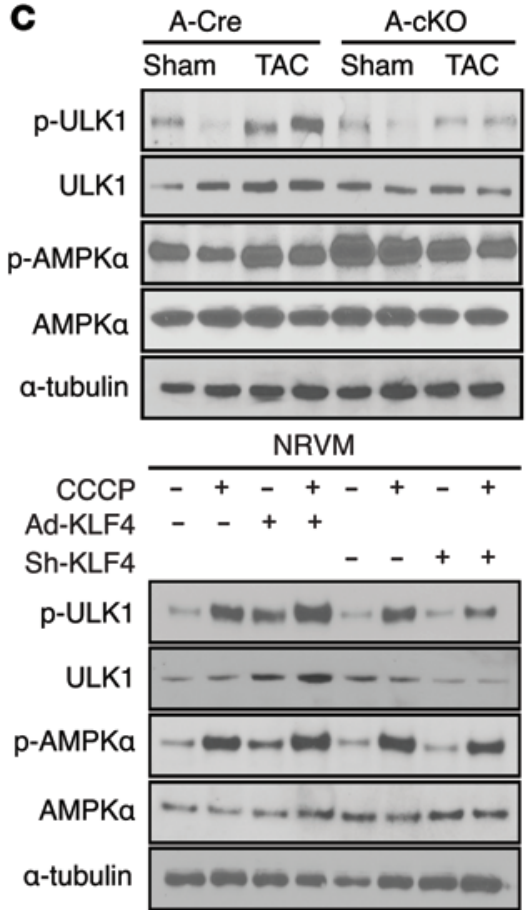

E

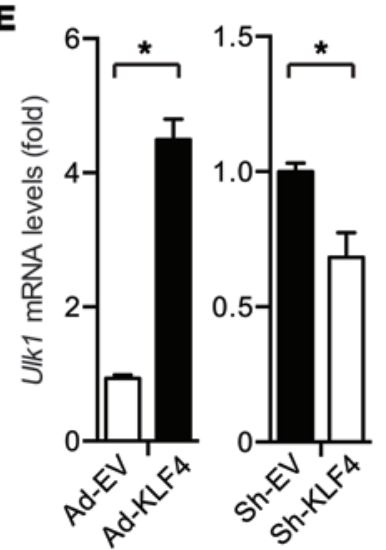

H


F

rat Ulk1 promoter

(1071bp)

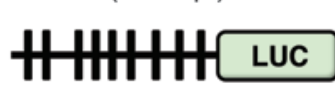

HHH Luc

Hth L

LUC
Ulk1 promoter activity (fold)

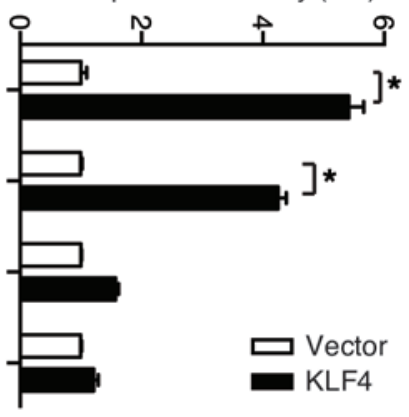

Figure 8. KLF4 regulates autophagy in the heart. (A) Western blot analysis for LC3 activation (LC3-I to LC3-II conversion). (B) Activation of the PINK1/ parkin pathway in the heart. A sample from MG132-treated cells was included as a positive control. Mitochondrial membrane protein TOM20 was used as loading control. (C) Activation of ULK1 signaling in the heart (top) and NRVMs (bottom). p-ULK1, p-Ser555. Heart samples were harvested at 3 days after surgery. NRVMs were infected with indicated adenovirus for 72 hours, followed by treatment with $20 \mu \mathrm{mol} / \mathrm{I}$ CCCP with or without $100 \mathrm{nmol} / \mathrm{I}$ BFA for 2 hours. (D) KLF4-induced autophagy flux is dependent on ULK1. (E) Expression of UIk1 mRNA in NRVMs with overexpression or knockdown of KLF4. (F) Ulk1 promoter truncation analysis. (G) Recruitment of KLF4 onto Ulk1 proximal promoter region. Arrowheads indicate PCR regions in ChIP assays. (H and I) Autophagy pathway qPCR array analysis. $n=3(E-I) .{ }^{*} P<0.05$, Student's $t$ test with Bonferroni correction. 


\section{KLF4 regulates mitochondrial homeostasis}



Figure 9. Model of KLF4's role in regulation of metabolic and mitochondrial genes. KLF4 serves as a critical transcriptional regulator of mitochondrial homeostasis in the heart through (i) cooperation with the ERR/PGC-1 transcriptional module on the regulation of metabolic and mitochondrial genes and (ii) direct regulation of the autophagy genes independent of the ERR/PGC-1 module. $X$, other transcription factors.

altered gene expression and mitochondrial biogenesis. Finally, KLF4 is found to bind to and synergize with ERR/PGC-1 on the promoters of common targets. These observations add to a growing appreciation that nuclear receptors and KLF crosstalk can orchestrate key cellular events. For example, in adipocytes, KLF5 cooperates with PPAR $\delta$ to induce transcription of the Pparg2 gene and, in doing so, promotes adipogenesis $(21,41)$. In cardiomyocytes, KLF15 cooperates with PPAR $\alpha$ in regulating lipid metabolism (42). Conversely, activation of GR signaling can induce a number of KLFs (e.g., KLF9, KLF11, and KLF15) in specific physiologic or pathologic contexts (43-45). Finally, the current study illuminates the importance of cooperativity between KLFs and nuclear receptors at two levels: (a) direct cooperation with the ERR/PGC-1 module and (b) upstream regulation of PPAR $\alpha$.

Another important aspect of the current work relates to KLF4 control of autophagy. This process is critical to heart homeostasis, and its disruption leads to a deterioration of cardiac function (10, 35). Importantly, much of the work to date has focused on cytoplasmic events that culminate in the autophagic response. In addition, a small but burgeoning literature underscores the importance of transcriptional regulation of this process (19), and our work supports and advances this view. Specifically, we show that KLF4 regulates numerous targets within the autophagy pathway, many of which (27 of 36 induced; 27 of 54 reduced) appear to be direct transcriptional targets (Figure 8G). We focused on Ulk1 (and its family member Ulk2), the mammalian homolog of ATG1, as it serves as a nodal point in the control of autophagy (19). We confirmed that both Ulk1 and Ulk2 are direct targets of KLF4 and are requisite for KLF4-induced autophagy. Previous studies have also indicated that additional transcription factors are involved in autophagy, including p53, NF- $\mathrm{kB}$, FOXO, and HIF-1 (19). Interestingly, work in different cellular contexts has revealed crosstalk between KLF4 and many of these other transcription factors, raising the possibility that KLF4 can also interact with these pathways in the transcriptional regulation of autophagy (46-49). Importantly, we believe that the KLF4-mediated induction of autophagy genes is likely independent of the ERR/PGC-1 module. Previous work suggests that PGC-1 can suppress autophagy in skeletal muscle (17), findings that we have confirmed in NRVMs as well (data not shown). While additional studies are warranted, our current observations suggest that KLF4 confers regulation on the autophagic pathway at many steps and hence functions as a transcriptional switch of autophagy. It has been shown that autophagy is required for heart health in the basal state and in response to stress $(9,10)$. Therefore, the impaired autophagy in KLF4-defecient heart may contribute to the ultimate cardiomyopathy.

There are several aspects of KLF4 in cardiomyocyte/mitochondrial biology that remain incompletely understood and will require further investigation. For example, while we observed a reduction in multiple lipid flux targets and complex I function at baseline in the KLF4-null hearts, cardiac function was preserved. It is possible that there is a certain threshold that was not crossed in the KLF4-deficient state at baseline that was crossed following stress (pressure overload and aging). Functional redundancy is another possibility, as other KLFs have been identified in the heart. Interestingly, a similar scenario has been observed in single Ppargcla KO, Ppargc1b KO, and Esrra $\mathrm{KO}$ mice. In all 3 cases, the mutant hearts were not distinguishable from control hearts at baseline but exhibited an enhanced susceptibility to cardiac dysfunction in response to stress $(13,30$, 50). However, compound KO mice with deficiency of both Ppargcla and Ppargc1b in the heart exhibited profound cardiac defects, supporting the idea of redundancy $(5,14)$. And finally, we note that the mitochondrial homeostasis consists not only of biogenesis and fission and fusion, but also of elimination of damaged mitochondria by autophagy. Whether KLF4 or the KLF4/ERR/PGC-1 pathway is operative in these processes to coordinate the entire mitochondrial life cycle is an important unanswered question.

This work adds significantly to the current hypothesis that KLFs are nodal regulators of cellular metabolism. The initial link was provided by Gray and colleagues, who showed that KLF15 regulates glucose metabolism (51). Since then, 8 additional members of this gene family have been shown to regulate adipogenesis both in mammals and worms $(52,53)$. Several KLF factors (e.g., KLF10, KLF11, KLF15) have been shown to regulate hepatic metabolism $(22,54-56)$. KLF15 has also been shown to control lipid flux and metabolism in cardiac and skeletal muscles $(57,58)$. However, a role for any member of this gene family as a central regulator of mitochondrial biology has not yet been reported, and thus, the current work serves as an important advance in this field.

\section{Methods}

Animal models. Mice with cardiac-specific postnatal deletion of Klf4 (Myh6-Cre Klf4 $4^{\sharp / l}$ mice, A-cKO mice) have been described previously (24). Mice with embryonic cardiac-specific deletion of Klf4 (E-cKO mice) were established by crossing a 2.8-kb Tagln promoter-driven Cre line to Klff $4^{\sharp / f l}$ mice (28). All mice are on a C57BL/6J background. Mice were housed in a temperature- and humidity-controlled specific pathogenfree facility with a 12-hour-light/dark cycle and ad libitum access to water and standard laboratory rodent chow. TAC model, echocardiography, and telemetry ECG were performed as described previously $(24,59)$.

Histology. Tissue samples were fixed in $10 \%$ neutralized formalin and embedded with paraffin following standard protocols. H\&E staining and trichrome staining were performed as described previously (24). Myocardial capillary density was revealed by CD31 (Mil- 
lipore, CBL1337) or CD34 staining (BD Pharmingen, 553731) (60, 61). DHE and EF5 staining were performed with fresh frozen sections in argon-buffered oxygen-free chambers $(62,63)$. Myocardial glycogen content was detected by a Periodic Acid-Schiff Kit (Sigma-Aldrich, 395B-1KT) using paraffin-embedded sections. Microscopic images were analyzed using ImagePro software for quantification.

Cell culture and cell-based assay. NRVMs (isolated from 2-day-old Sprague Dawley rats) and HEK293 cells (ATCC CRL-1573) were cultured as described previously (24). Expression plasmids (KLF4, ERR $\alpha$, ERR $\gamma$, PGC- $1 \alpha$, and PGC-1 $\beta$ ), adenoviral KLF4, and PPAR $\alpha$ and PDK4 promoter luciferase constructs have been described previously (24, 31-33). A shRNA targeting rat Klf4 (GTATGTGCCCCAAGATTAA) was designed and packaged into adenovirus by Welgen Inc. Adenoviral shRNA targeting mouse/rat Ulk1 and Ulk2 was purchased from Vector Biolabs (shADV-275618 and shADV-275619). The promoter regions of mouse Cycs (1,766 bp) and rat Ulk1 (1,071 bp) were amplified by PCR and cloned into pGL3-basic luciferase vector. Mutations in promoters were introduced by site-directed mutagenesis with a QuikChange Lightning Site-Directed Mutagenesis Kit (Stratagene, 210518). All plasmids were sequenced to ensure sequence accuracy. Adenoviral infection was carried out with indicated virus (10 MOI for overexpression or $50 \mathrm{MOI}$ for knockdown) for 48 to 72 hours prior to experiments. Transient transfections were carried out using the X-tremeGENE HP DNA Transfection Reagent (Roche, 06366236001). Luciferase-generated bioluminescence was recorded on a Veritas Luminometer (Turner Biosystems) 24 hours after transfection (24). FAO rate was determined by release of $\left[{ }^{3} \mathrm{H}\right]-\mathrm{H}_{2} \mathrm{O}$ in the aqueous phase of cell lysate chloroform extracts from oxidation of $1-\left[{ }^{3} \mathrm{H}\right]$-oleic acid as described previously (47). Glycolysis rate and mitochondrial respiration rate in NRVMs were assessed using a Seahorse XFp Extracellular Flux Analyzer with the XFp Glycolysis Stress Test Kit and the XFp Cell Mito Stress Test Kit, respectively (Seahorse Bioscience) (42). For in vitro autophagy studies, NRVMs were infected with indicated adenovirus for 72 hours, followed by treatment with $20 \mu \mathrm{mol} / \mathrm{l} \mathrm{CCCP}$ with or without $100 \mathrm{nmol} / \mathrm{l} \mathrm{BFA}$ for 2 hours (35) (CCCP, Sigma-Aldrich, C2759; BFA, Sigma-Aldrich, B1793).

RNA extraction and $q P C R$. Tissue samples were homogenized in TRIzol reagent (Life Technologies, 15596-026) with a TissueLyser (Qiagen). Cell samples were directly dissolved in TRIzol reagent. Total RNA was extracted, treated with DNase I (Life Technologies, 18068015), purified using the RNeasy MinElute Cleanup Kit (Qiagen, 74204), and reverse transcribed to complementary DNA using the iScript Reverse Transcription Kit (Bio-Rad, 170-8841). qPCR was performed with either the TaqMan method (Roche Universal ProbeLibrary System) or the SYBR green method on a ViiA 7 Real-Time PCR System (Applied Biosystems). Relative expression was calculated using the $\Delta \Delta \mathrm{Ct}$ method with normalization to Gapdh. PCR primer sequences are listed in Supplemental Table 2.

Mitochondrial respiration studies. Myocardial mitochondria of 4 individual mice were pooled for each experiment. In brief, ventricles were excised, pooled, and washed in ice-cold Chappell-Perry buffer, followed by homogenization. Interfibrillar mitochondria were isolated using differential centrifugation in combination with trypsin digestion (64). Mitochondrial respiration rates were assessed by measuring oxygen consumption with a Clark-type oxygen electrode at $30^{\circ} \mathrm{C}$. After depletion of endogenous substrates with $100 \mu \mathrm{mol} / \mathrm{l} \mathrm{ADP}$, a state 3 respiration rate was recorded in the presence of $100 \mu \mathrm{mol} / \mathrm{l} \mathrm{ADP}$ and a state 4 respiration rate was recorded after ADP depletion. ETC enzyme activities were measured spectrophotometrically as specific donor-acceptor oxidoreductase activities using permeabilized mitochondria (64).

Mitochondrial genome quantification. Total DNA was extracted from the hearts or NRVMs using the QIAamp DNA Mini Kit (Qiagen, 51304). Mitochondrial DNA content was assessed by qPCR using primers specific for multiple mitochondrial-encoded genes ( $m t-\mathrm{Col}$, $m t-C o 2, m t-N d 1, m t-N d 5, m t-C y t b, m t-A t p 6)$ and normalized to nuclear DNA content (a specific locus on mouse chromosome 6 or rat chromosome 4) using the $\Delta \Delta \mathrm{Ct}$ method. Primer sequences are listed in Supplemental Table 2.

Transmission electron microscopy. Small pieces of tissue from the LV free wall were fixed by sequential immersion in triple aldehyde-DMSO, ferrocyanide-reduced osmium tetroxide, and acidified uranyl acetate; dehydrated in ascending concentrations of ethanol; passed through propylene oxide; and embedded in Poly/Bed resin (Polysciences Inc., 21844-1). Thin sections were sequentially stained with acidified uranyl acetate, followed by a modification of Sato's triple lead stain, and examined with a JEOL 1200EX electron microscope (65).

Western blot. Protein from cultured cells or tissues was extracted using RIPA buffer (Sigma-Aldrich, R0278) supplemented with proteinase and phosphatase inhibitor cocktails (Roche, 4693132001 and 4906845001). Mitochondrial protein was solubilized with 1\% DDM (Life Technologies, BN2005) or 2\% CHAPS (Sigma-Aldrich, C5070). The following antibodies were used: phospho-AMPK $\alpha$ (Thr172) (Cell Signaling, 2535), AMPK $\alpha$ (Cell Signaling, 2603), $\alpha$-tubulin (Sigma-Aldrich, T9026), mt-CO1 (Abcam, ab14705), mt-CO2 (Abcam, ab110258), NDUFS1 (Abcam, ab169540), ATP5a (Abcam, ab14748), COX IV (Cell Signaling, 4844), KLF4 (Santa Cruz, sc-20691), LC3B (Cell Signaling, 3868), PINK1 (Sigma-Aldrich, SAB1304438), parkin (Cell Signaling, 4211), SQSTM1/p62 (Cell Signaling, 5114), TOM20 (Santa Cruz, sc-11415), ubiquitin (Cell Signaling, 3936), phospho-ULK1 (Ser555) (Cell Signaling, 5869), ULK1 (Cell Signaling, 8054), phospho-acetyl-CoA carboxylase (Ser79) (Cell Signaling, 11818), acetyl-CoA carboxylase (Cell Signaling, 3676), HRP-linked anti-FLAG (Sigma-Aldrich, A8592), HRPlinked anti-HA (Sigma-Aldrich, H6533), HRP-linked anti-rabbit IgG (Cell Signaling, 7074), and HRP-linked anti-mouse IgG (Cell Signaling, 7076). See complete unedited blots in the supplemental material.

Coimmunoprecipitation. Total protein from HEK293 cells was prepared using Pierce IP Lysis Buffer (Life Technologies, 87787) in combination with sonication to ensure extraction of nuclear proteins. For each immunoprecipitation, $1 \mathrm{mg}$ protein was immunoprecipitated with 2 to $5 \mu \mathrm{g}$ of antibody bound to agarose or magnetic beads. Anti-FLAG (EZview Red ANTI-FLAG M2 Affinity Gel, Sigma-Aldrich, F2426), antiHA (EZview Red Anti-HA Affinity Gel, Sigma-Aldrich, E6779), and anti-KLF4 (Santa Cruz, sc-20691) antibodies were used for immunoprecipitation. Immune complexes were washed extensively and eluted in Laemmli Sample Buffer (Bio-Rad, 161-0737). Precipitated and input proteins were subjected to SDS-PAGE and immunoblotted as indicated.

Gel filtration chromatography. Cell-free extract was concentrated to $<200 \mu$ l by ultrafiltration (total protein $\sim \mathrm{mg}$ ) and applied at a flow rate of $0.5 \mathrm{ml} / \mathrm{min}$ to a Superdex $20010-\times 300-\mathrm{mm}$ column equilibrated with PBS. The eluent was collected in $1 \mathrm{ml}$ fractions and concentrated to $40 \mu \mathrm{l}$ by ultrafiltration. Eluted fractions were analyzed by Western blotting. The molecular weight of proteins in the fractions was determined using the elution profile of gel-filtration standards (Bio-Rad, 151-1901). 
ChIP. NRVMs $\left(2 \times 10^{7}\right)$ were fixed with $1 \%$ formaldehyde, and chromatin was extracted and sonicated using a BioRuptor (Diagnode). The sonicated chromatin was immunoprecipitated with 2 to $5 \mu \mathrm{g}$ of anti-KLF4 antibodies (Santa Cruz, sc-20691) bound to Dynabeads (Life Technologies, 10003D), followed by extensive washing and elution. Chromatin was then reverse cross-linked, followed by purification of genomic DNA. Target and nontarget regions were amplified by qPCR in both the precipitated and input samples. A locus on rat chromosome 4 was used as a nontarget control. ChIP primer sequences are listed in Supplemental Table 2.

Microarray analysis. Total ventricular RNA was purified from A-Cre and A-cKO mice with sham surgery or TAC at 3 days after surgery ( $n=4$ in each group). Sample preparation, labeling, and array hybridizations were performed according to standard protocols from the UCSF Shared Microarray Core Facilities and Agilent Technologies (http://www.arrays.ucsf.edu and http://www.agilent.com). Total RNA quality was assessed using a Pico Chip on an Agilent 2100 Bioanalyzer (Agilent Technologies). RNA was amplified and labeled with Cy3-CTP using the Agilent Low RNA Input Fluorescent Linear Amplification Kit following the manufacturer's protocol. Labeled cRNA was assessed using the Nanodrop ND-100 (Nanodrop Technologies Inc.), and equal amounts of Cy3-labeled target were hybridized to Agilent whole mouse genome $4 \mathrm{x} 44 \mathrm{~K}$ Ink-jet arrays. Hybridizations were performed for 14 hours, according to the manufacturer's protocol. Arrays were scanned using the Agilent Microarray Scanner, and raw signal intensities were extracted with Feature Extraction v10.1 software (Agilent). Microarray data were log transformed before differential expression analysis using the limma package. Probes with a $P$ value of less than 0.05 after adjusting for false discovery rate using Benjamini and Hochberg method were considered significant in differential expression. Enrichment in biological processes was analyzed using the DAVID bioinformatics suite. The microarray data set has been deposited to the NCBI Gene Expression Omnibus, with the accession number GSE61177.
Statistics. Results are presented as mean \pm SEM. Two-tailed Student's $t$ test was used to compare the differences between 2 groups. One-way ANOVA with Bonferroni correction was used for multiple comparisons. Survival curves were compared using the log-rank test. Statistical significance was defined as $P<0.05$.

Study approval. All animal studies were approved by the Institutional Animal Care and Use Committee of Case Western Reserve University. The use of human samples was approved by the institutional review boards of Columbia University and Case Western Reserve University (IRB-AAAE7393). Human heart samples were obtained as described previously $(57,66)$.

\section{Acknowledgments}

We thank Xiaodong Bai (RNA Center at Case Western Reserve University) for microarray data analysis and Quan Chen (Institute of Zoology, Chinese Academy of Sciences, Beijing, China) for help on autophagy studies. This work was supported by an American Heart Association Established Investigator Award (to M.K. Jain); American Heart Association National Scientist Development grants 12SDG12070077 (to X. Liao) and 12SDG12050558 (to Y. Lu); and NIH grants R01HL058493 (to D.P. Kelly); R01HL110630-01, R01HL112486, R01HL086548, and R01HL119195 (to M.K. Jain); T32HL105338 and F32HL110538 (to D.A. Prosdocimo). This work was also generously supported by Tom F. Peterson (to M.K. Jain).

Address correspondence to: Mukesh K. Jain, Iris S. Bert L. Wolstein Research Building, 2103 Cornell Road, Room 4537, Cleveland, Ohio 44106-7290, USA. Phone: 216.368.3607; E-mail: mukesh.jain2@case.edu. Or to: Xudong Liao, 2103 Cornell Road, WRB-4503, Cleveland, Ohio 44106, USA. Phone: 216.368.0591; E-mail: xudong.liao@case.edu.
1. Lopaschuk GD, Ussher JR, Folmes CD, Jaswal JS, Stanley WC. Myocardial fatty acid metabolism in health and disease. Physiol Rev. 2010;90(1):207-258.

2. Barth E, Stammler G, Speiser B, Schaper J. Ultrastructural quantitation of mitochondria and myofilaments in cardiac muscle from 10 different animal species including man. J Mol Cell Cardiol. 1992;24(7):669-681.

3. Lesnefsky EJ, Moghaddas S, Tandler B, Kerner J, Hoppel CL. Mitochondrial dysfunction in cardiac disease: ischemia--reperfusion, aging, and heart failure. J Mol Cell Cardiol. 2001;33(6):1065-1089.

4. Girard J, Ferre P, Pegorier JP, Duee PH. Adaptations of glucose and fatty acid metabolism during perinatal period and suckling-weaning transition. Physiol Rev. 1992;72(2):507-562.

5. Lai L, et al. Transcriptional coactivators PGC-1alpha and PGC-lbeta control overlapping programs required for perinatal maturation of the heart. Genes Dev. 2008;22(14):1948-1961.

6. Youle RJ, van der Bliek AM. Mitochondrial fission, fusion, and stress. Science. 2012;337(6098):1062-1065.

7. Kubli DA, Gustafsson AB. Mitochondria and mitophagy: the yin and yang of cell death control. Circ Res. 2012;111(9):1208-1221.

8. Chen Y, Liu Y, Dorn GW. Mitochondrial fusion is essential for organelle function and cardiac homeostasis. Circ Res. 2011;109(12):1327-1331.

9. Kuma A, et al. The role of autophagy during the early neonatal starvation period. Nature. 2004;432(7020):1032-1036.

10. Nakai A, et al. The role of autophagy in cardiomyocytes in the basal state and in response to hemodynamic stress. Nat Med. 2007;13(5):619-624.

11. Alaynick WA, et al. ERRgamma directs and maintains the transition to oxidative metabolism in the postnatal heart. Cell Metab. 2007;6(1):13-24.

12. Dufour CR, et al. Genome-wide orchestration of cardiac functions by the orphan nuclear receptors ERR $\alpha$ and $\gamma$. Cell Metab. 2007;5(5):345-356.

13. Huss JM, et al. The nuclear receptor ERR $\alpha$ is required for the bioenergetic and functional adaptation to cardiac pressure overload. Cell Metab. 2007;6(1):25-37.

14. Martin OJ, et al. A role for peroxisome proliferator-activated receptor $\gamma$ coactivator- 1 in the control of mitochondrial dynamics during postnatal cardiac growth. Circ Res. 2014;114(4):626-636.

15. Arany Z, et al. Transcriptional coactivator PGC- $1 \alpha$ controls the energy state and contractile function of cardiac muscle. Cell Metab. 2005;1(4):259-271.

16. Huss JM, Kelly DP. Nuclear receptor signaling and cardiac energetics. Circ Res.
2004;95(6):568-578

17. Wenz T, Rossi SG, Rotundo RL, Spiegelman BM, Moraes CT. Increased muscle PGC-1 $\alpha$ expression protects from sarcopenia and metabolic disease during aging. Proc Natl Acad Sci U S A. 2009;106(48):20405-20410.

18. Vainshtein A, Desjardins EM, Armani A, Sandri M, Hood DA. PGC- $1 \alpha$ modulates denervationinduced mitophagy in skeletal muscle. Skelet Muscle. 2015;5:9.

19. Fullgrabe J, Klionsky DJ, Joseph B. The return of the nucleus: transcriptional and epigenetic control of autophagy. Nat Rev Mol Cell Biol. 2014;15(1):65-74.

20. McConnell BB, Yang VW. Mammalian Kruppellike factors in health and diseases. Physiol Rev. 2010;90(4):1337-1381.

21. Oishi Y, et al. Kruppel-like transcription factor KLF5 is a key regulator of adipocyte differentiation. Cell Metab. 2005;1(1):27-39.

22. Gray S, et al. Regulation of gluconeogenesis by Kruppel-like factor 15. Cell Metab. 2007;5(4):305-312.

23. Papait R, et al. Genome-wide analysis of histone marks identifying an epigenetic signature of promoters and enhancers underlying cardiac hypertrophy. Proc Natl Acad Sci U S A. 2013;110(50):20164-20169. 
24. Liao X, et al. Kruppel-like factor 4 regulates pressure-induced cardiac hypertrophy. J Mol Cell Cardiol. 2010;49(2):334-338.

25. Dorn GW. Mitochondrial dynamics in heart disease. Biochim Biophys Acta. 2013;1833(1):233-241.

26. Lehman JJ, Barger PM, Kovacs A, Saffitz JE, Medeiros DM, Kelly DP. Peroxisome proliferator-activated receptor $\gamma$ coactivator-1 promotes cardiac mitochondrial biogenesis. JClin Invest. 2000;106(7):847-856

27. Maillet M, et al. Heart-specific deletion of CnB1 reveals multiple mechanisms whereby calcineurin regulates cardiac growth and function. J Biol Chem. 2010;285(9):6716-6724.

28. Lepore JJ, Cheng L, Min Lu M, Mericko PA, Morrisey EE, Parmacek MS. High-efficiency somatic mutagenesis in smooth muscle cells and cardiac myocytes in SM22 $\alpha$-Cre transgenic mice. Genesis. 2005;41(4):179-184.

29. Yoshida T, et al. Smooth and cardiac muscle-selective knock-out of Kruppel-like factor 4 causes postnatal death and growth retardation. J Biol Chem. 2010;285(27):21175-21184.

30. Arany Z, Novikov M, Chin S, Ma Y, Rosenzweig A, Spiegelman BM. Transverse aortic constriction leads to accelerated heart failure in mice lacking PPAR- $\gamma$ coactivator $1 \alpha$. Proc Natl Acad Sci U S A. 2006;103(26):10086-10091.

31. Huss JM, Torra IP, Staels B, Giguere V, Kelly DP. Estrogen-related receptor $\alpha$ directs peroxisome proliferator-activated receptor alpha signaling in the transcriptional control of energy metabolism in cardiac and skeletal muscle. Mol Cell Biol. 2004;24(20):9079-9091.

32. Wende AR, Huss JM, Schaeffer PJ, Giguere V, Kelly DP. PGC- $1 \alpha$ coactivates PDK4 gene expression via the orphan nuclear receptor ERR $\alpha$ : a mechanism for transcriptional control of muscle glucose metabolism. Mol Cell Biol. 2005;25(24):10684-10694.

33. Huss JM, Kopp RP, Kelly DP. Peroxisome proliferator-activated receptor coactivator-1alpha (PGC-1 $\alpha$ ) coactivates the cardiac-enriched nuclear receptors estrogen-related receptor- $\alpha$ and $-\gamma$. Identification of novel leucine-rich interaction motif within PGC-1 $\alpha$. J Biol Chem. 2002;277(43):40265-40274.

34. Finck BN, Kelly DP. PGC-1 coactivators: inducible regulators of energy metabolism in health and disease. J Clin Invest. 2006;116(3):615-622.

35. Oka T, et al. Mitochondrial DNA that escapes from autophagy causes inflammation and heart failure. Nature. 2012;485(7397):251-255.

36. Chen Y, Dorn GW. PINK1-phosphorylated mito- fusin 2 is a Parkin receptor for culling damaged mitochondria. Science. 2013;340(6131):471-475.

37. Nakatogawa H, Suzuki K, Kamada Y, Ohsumi Y. Dynamics and diversity in autophagy mechanisms: lessons from yeast. Nat Rev Mol Cell Biol. 2009;10(7):458-467.

38. Lee EJ, Tournier C. The requirement of uncoordinated 51-like kinase 1 (ULK1) and ULK2 in the regulation of autophagy. Autophagy. 2011;7(7):689-695.

39. Egan DF, et al. Phosphorylation of ULK1 (hATG1) by AMP-activated protein kinase connects energy sensing to mitophagy. Science. 2011;331(6016):456-461.

40. Kim J, Kundu M, Viollet B, Guan KL. AMPK and $\mathrm{mTOR}$ regulate autophagy through direct phosphorylation of Ulk1. Nat Cell Biol. 2011;13(2):132-141.

41. Oishi Y, et al. SUMOylation of Kruppel-like transcription factor 5 acts as a molecular switch in transcriptional programs of lipid metabolism involving PPAR- $\delta$. Nat Med. 2008;14(6):656-666.

42. Prosdocimo DA, et al. KLF15 and PPAR $\alpha$ cooperate to regulate cardiomyocyte lipid gene expression and oxidation. PPAR Res. 2015;2015:201625

43. Masuno K, et al. Expression profiling identifies Klf15 as a glucocorticoid target that regulates airway hyperresponsiveness. Am J Respir Cell Mo Biol. 2011;45(3):642-649.

44. Sasse SK, et al. The glucocorticoid receptor and KLF15 regulate gene expression dynamics and integrate signals through feed-forward circuitry. Mol Cell Biol. 2013;33(11):2104-2115.

45. Grunewald M, et al. Mechanistic role for a novel glucocorticoid-KLF11 (TIEG2) protein pathway in stress-induced monoamine oxidase A expression. J Biol Chem. 2012;287(29):24195-24206.

46. Yusuf I, et al. KLF4 is a FOXO target gene that suppresses B cell proliferation. Int Immunol. 2008;20(5):671-681.

47. Liao X, et al. Kruppel-like factor 4 regulates macrophage polarization. JClin Invest. 2011;121(7):2736-2749.

48. Rowland BD, Bernards R, Peeper DS. The KLF4 tumour suppressor is a transcriptional repressor of p53 that acts as a context-dependent oncogene. Nat Cell Biol. 2005;7(11):1074-1082.

49. Mathieu J, et al. HIF induces human embryonic stem cell markers in cancer cells. Cancer Res. 2011;71(13):4640-4652.

50 . Riehle C, et al. PGC-1 $\beta$ deficiency accelerates the transition to heart failure in pressure overload hypertrophy. Circ Res. 2011;109(7):783-793.

51. Gray S, et al. The Kruppel-like factor KLF15 reg- ulates the insulin-sensitive glucose transporter GLUT4. J Biol Chem. 2002;277(37):34322-34328.

52. Hashmi S, Zhang J, Siddiqui SS, Parhar RS, Bakheet R, Al-Mohanna F. Partner in fat metabolism: role of KLFs in fat burning and reproductive behavior. 3 Biotech. 2011;1(2):59-72.

53. Lee H, et al. Kruppel-like factor KLF8 plays a critical role in adipocyte differentiation. PLoS One. 2012;7(12):e52474.

54. Guillaumond F, et al. Kruppel-like factor KLF10 is a link between the circadian clock and metabolism in liver. Mol Cell Biol. 2010;30(12):3059-3070.

55. Zhang $\mathrm{H}$, et al. Mouse KLF11 regulates hepatic lipid metabolism. J Hepatol. 2013;58(4):763-770.

56 . Jeyaraj D, et al. Klf15 orchestrates circadian nitrogen homeostasis. Cell Metab. 2012;15(3):311-323.

57. Prosdocimo DA, et al. Kruppel-like factor 15 is a critical regulator of cardiac lipid metabolism. J Biol Chem. 2014;289(9):5914-5924.

58. Haldar SM, et al. Kruppel-like factor 15 regulates skeletal muscle lipid flux and exercise adaptation. Proc Natl Acad Sci U S A. 2012;109(17):6739-6744.

59. Jeyaraj D, et al. Circadian rhythms govern cardiac repolarization and arrhythmogenesis. Nature. 2012;483(7387):96-99.

60. Hale AT, et al. Endothelial Kruppel-like factor 4 regulates angiogenesis and the Notch signaling pathway. J Biol Chem. 2014;289(17):12016-12028.

61. Pusztaszeri MP, Seelentag W, Bosman FT. Immunohistochemical expression of endothelial markers CD31, CD34, von Willebrand factor, and Fli-1 in normal human tissues. J Histochem Cytochem. 2006;54(4):385-395.

62. Wikenheiser J, Doughman YQ, Fisher SA, Watanabe M. Differential levels of tissue hypoxia in the developing chicken heart. Dev Dyn. 2006;235(1):115-123.

63. Adams GN, et al. Murine prolylcarboxypeptidase depletion induces vascular dysfunction with hypertension and faster arterial thrombosis. Blood. 2011;117(14):3929-3937.

64. Rosca MG, et al. Cardiac mitochondria in heart failure: decrease in respirasomes and oxidative phosphorylation. Cardiovasc Res. 2008;80(1):30-39.

65. Fujioka H, Tandler B, Hoppel CL. Mitochondrial division in rat cardiomyocytes: an electron microscope study. Anat Rec (Hoboken). 2012;295(9):1455-1461.

66. Chokshi A, et al. Ventricular assist device implantation corrects myocardial lipotoxicity, reverses insulin resistance, and normalizes cardiac metabolism in patients with advanced heart failure. Circulation. 2012;125(23):2844-2853. 